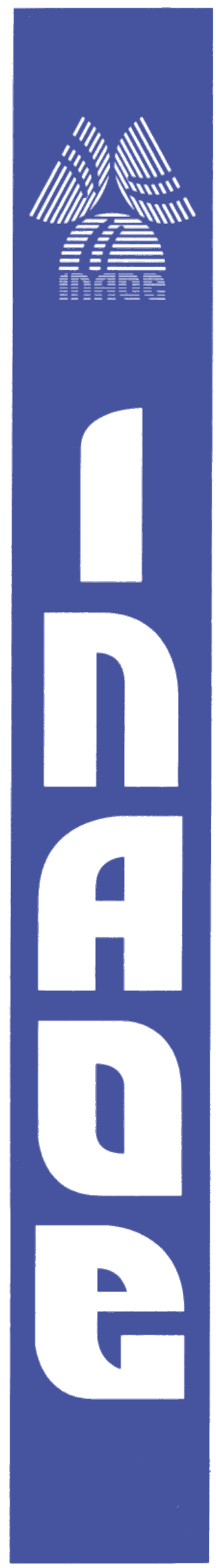

\title{
Nonlinear Pulse Propagation inside Coupled Silicon Nanowires
}

by

\author{
Phys. Néstor Lozano Crisóstomo \\ INAOE
}

Thesis submitted as partial requirement for the degree of

\section{MASTER IN SCIENCES WITH SPECIALITY OF OPTICS}

at

Instituto Nacional de Astrofísica Óptica y Electrónica August 2011

Tonantzintla, Puebla.

Supervised by:

Dr. José Javier Sánchez Mondragón

Optics Department

INAOE

and

Professor Govind P. Agrawal

The Institute of Optics

University of Rochester

CINAOE 2011 All right reserved

The autor gives permission to INAOE to reproduce and distribute copies of this thesis in whole or in part.

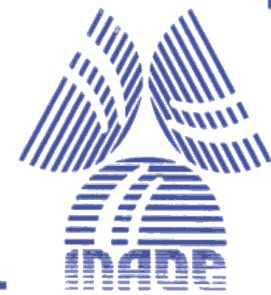




\section{Abstract}

Title of dissertation:

Nonlinear Pulse Propagation inside Coupled Silicon Nanowires Néstor Lozano Crisóstomo, Master in Optics, 2011

Dissertation directed by:

\section{Dr. José Javier Sánchez Mondragón}

Optics Department, INAOE and

\section{Professor Govind P. Agrawal}

The institute of Optics, University of Rochester

This thesis investigates nonlinear effects in semiconductor nanowires for optical switching. In this work we describe the nonlinear propagation of optical pulses through an array of silicon nanowires made with the silicon-oninsulator (SOI) technology. A generalized analysis of the nonlinear coupled system is given, where we have considered the vector nature of optical modes and the effects of two-photon absorption (TPA) on various nonlinear processes. The general theoretical model includes not only the effects of freecarrier absorption (FCA) and free-carrier dispersion (FCD), but also linear and nonlinear losses, and it extends previous vector nonlinear models to the case where coupling of supermodes of a waveguide array occurs in silicon waveguides. Analytical solutions are provided for the coupled-mode equations, in some cases in which the density of the free carrier is relatively low, and dispersive effects are relatively weak, assuming that the nonlinear effects do not affect the waveguide modes significantly. The impact of twophoton absorption and free-carriers effect on the properties of the nonlinear coupling effects is studied in detail; together with the evolution of optical power inside an array of short silicon nanowires. 


\section{Resumen}

Título de la tesis:

Propagación No Lineal de Pulsos en Nanoguias de Silicio Acopladas Néstor Lozano Crisóstomo, Maestría en Óptica, 2011

Tesis dirigida por:

\section{Dr. José Javier Sánchez Mondragón}

Departamento de Óptica, INAOE $y$

\section{Professor Govind P. Agrawal}

The institute of Optics, University of Rochester

En esta tesis se investigan los efectos no lineales en nanoguías de onda semiconductoras para su implementación al switching óptico. En este trabajo se describe la propagación no lineal de pulsos ópticos a través de un arreglo de nanoguias de silicio hechas con la tecnología SOI. Se presenta un análisis generalizado del sistema no lineal acoplado donde se considera la naturaleza vectorial de los modos ópticos y los efectos de la absorción de dos fotones sobre varios procesos no lineales. El modelo teórico general incluye no solo los efectos de la absorción y dispersión causados por los cargadores libres, sino también las pérdidas lineales y no lineales, además de que se extienden los modelos teóricos anteriores al caso donde el acoplamiento de supermodos de un arreglo de guías ocurre. Se muestran soluciones analíticas a las ecuaciones de modos acoplados para los casos en donde la densidad de las cargas libres es relativamente baja, y los efectos dispersivos son relativamente débiles, asumiendo que los efectos no lineales no afectan a los modos significativamente. Se estudia el impacto de la absorción de dos fotónes y el efecto de las cargas libres sobre las propiedades de los efectos de acoplamiento, junto con la evolución del campo dentro del arreglo. 


\section{Acknowledgements}

This dissertation would not have been possible without the guidance and help of several individuals who in one way or other contributed and extended their valuable assistance in the preparation and completion of this study. First and foremost, I am heartily thankful to my advisor and friend, Dr. José Javier Sanchez Mondragón for his invaluable support, advice, recommendation, patience, and encouragement. He has made available his support in a number of ways. I have learned a lot of from him, above all things the manner to face the world. His sincerity, dedication and friendship I will never forget.

It is an honor for me to thank Professor Govind P. Agrawal for his considerable support, guidance, supervision and assistant, for the insights he shared me and for patiently answering a great many questions. I owe my deepest gratitude to him for letting me have a good time with his research group. He made me be part of his research group in all ways. I have learned a lot of from him.

I would like to express my gratitude to all those people who have helped and inspired me throughout my master study. Special thanks to Brian Daniel for his assistant, unselfish and unfailing support during my internship at the Institute of Optics, University of Rochester, but mostly for his friendship. Many thanks to Omar Santiago and Paloma Ibarra for their help, friendship and fun. I would like to thank Mrs. Gina Kern for her friendly support and consideration regarding my internship requirements. She was always very helpful, friendly and nice. I am very grateful for her considerations. Also, I would like to express my most sincere gratitude to my former Rector Mrs. 
Nora Claudia Lusting for their trust in my hometown youth and by creating the opportunity for my educational experience.

I would like to express my gratitude to the National Council for Science and Technology (CONACYT) for its considerable financial support through the postgraduate scholarship with number 235214 and a Beca Mixta for the internship during which most of this thesis work was carried out. Also, I am also grateful to CONACYT for the research grant CB-2008/101378 that provides the support for the general project where this thesis is included. I am aware that all these supports meant the work and interest of people and to them my warmest gratitude for their interest in our success. They are too many to mention and to be aware of, but in their name let me thank to Mrs. Gabriela Gómez Gutiérrez for my Beca Mixta and those of my classmates, to Q. Regina M. Alarcón Contreras for the funding for the marvelous Photonic Workshop where I managed to meet Prof. Agrawal and to Biol. Martha Patricia Ojeda Carrasco for helping us with our project; to all of you thank you very much.

Lastly, I offer my regards and thanks to all of those who supported me in any respect during the completion of the project.

\section{Néstor Lozano-Crisóstomo}




\section{Dedication}

To my grandparents Isidora and Juan.

To Nataly and Néstor

And to my mother Maria Luisa 


\section{Table of Contents}

Abstract i

Acknowledgements iii

Dedications v v

1 Introduction 1

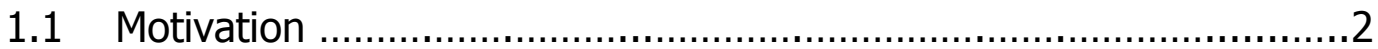

1.2 SOI Waveguides Characteristics ............................................. 4

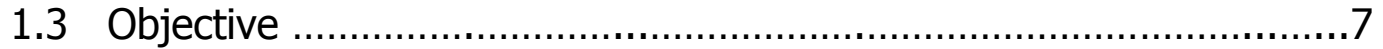

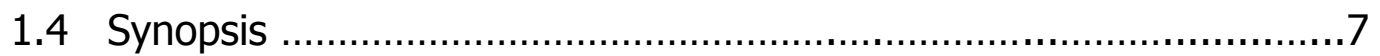

2 Novel Properties of SOI Waveguides 10

2.1 Dispersion in SOI Waveguides .................................................10

2.1.1 Material dispersion of silicon ........................................11

2.1.2 Engineered dispersion ................................................12

2.2 Geometric Dependence of Nonlinear Parameters ..........................13

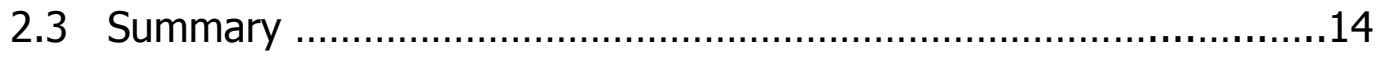

3 Theoretical Framework 16

3.1 The Wave Equation for Silicon ..................................................16

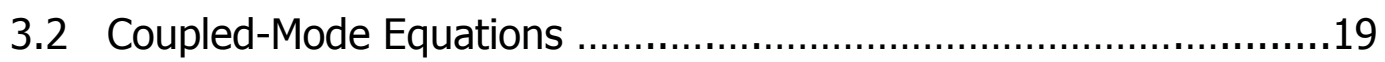

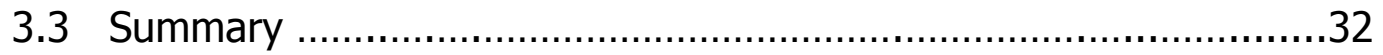

4 Nonlinear Propagation in Coupled Silicon Nanowires 35

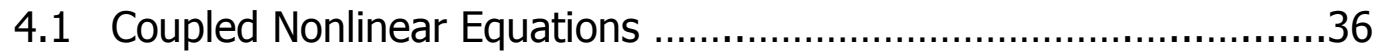

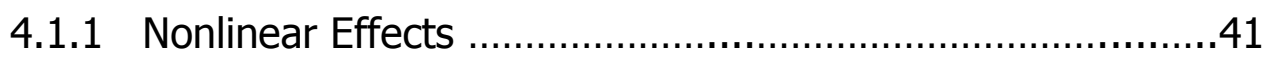

4.1.2 Free-Carrier Effects …..................................................

4.1.3 Dispersion Effects ........................................................... 


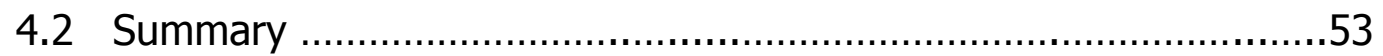

5 Conclusions $\quad 55$

Appendix A $\quad 57$

$\begin{array}{ll}\text { List of Figures } & 60\end{array}$ 


\section{Chapter 1}

\section{Introduction}

This thesis basically deals with the nonlinear propagation of optical pulses through a pair of coupled silicon waveguides at nanoscale dimensions. This is the study of various effects and phenomena related to the interaction of intense coherent light with matter, and its optical applications. Essentially, the topic of this work is nonlinear optics. This work involves an analytical analysis and modeling of the nonlinear propagation of soliton pulses in an array of novel nano-scale single-mode SOI rib waveguides. For applications, it is of particular importance the field of high-speed silicon photonic switches for a ultrahigh information processing in optical on-chip networks. In fact, the silicon all-optical switch could offer advantages for silicon interconnects and components. Optical signal processing is often based on ultrafast nonlinear effects in waveguide devices. To simplify, a structure that consist of only two coupled silicon nanowires is used in order to obtain the input-output power relations of optical pulses taking into account the linear losses, the Kerr nonlinearity, TPA, FCA, FCD, linear coupling and the power-dependent coupling effect, and implement them to the optical switching. However, the general modeling was made for an array of several silicon nanowires in close proximity to each other. In general, this report shows, in one way, how ultrafast nonlinear effects in silicon waveguide devices as the silicon photonic switch lead to the optical signal processing on high-speed optical networks. 
Due to the tight confinement and high-power input signals, nonlinearities and free-carrier effects in the properties of silicon waveguides are often of significant relevance for the technological applications, sometimes useful, sometimes hampering. To understand these nonlinearities and free-carriers effects, and to use them for applications, will be of basic importance for the further development of photonic applications.

\subsection{Motivation}

Silicon photonics has aroused an increasing interest in recent years at international level $[1,2]$. Its potential applications on the photonics industry have attracted much attention because the recent progress on silicon-based photonics components, photonic integrated circuits, and optoelectronic integrated circuits [2]. Silicon photonics has played a steadily increasing role in technological innovations and it is the expected continuation to electronics. Silicon, in addition of being the most fundamental material in the $20^{\text {th }}$ century information technologies, it has become a Photonic material and therefore of special interest in modern information technologies. The most promising applications are in the fields of optical interconnects in complementary-metal-oxide-semiconductor (CMOS) integrated circuits and optical communications, where silicon can provide low cost solutions and technological innovations [3-4]. Silicon photonics is now the most active discipline within the field of integrated optics [5] which uses the SOI wafer technique and nano-scale photolithography to fabricate silicon photonic devices. The aim of the silicon photonics industry is to obtain waveguides, ultrafast modulators, switches, light sources, detectors, photonic crystals and plasmonic devices [1], and integrate all these components into a silicon photonic chip. If an integrated silicon photonic chip could be developed, it 
could bring significant, even revolutionary changes, to the next generation communications industry, by radically altering the price, power, and size for photonic components [3]. An efficient way to the optical integration of siliconbased photonic components is using processes similar to the baseline CMOS processes, for which one could achieve much higher yielding optical devices, like passive and active opto-electronic components at a lower cost [1-5].

Silicon is a well-understood and robust material. It exhibits very low optical losses (material losses $<0.01 \mathrm{~dB} / \mathrm{cm}$ losses in unintentionally doped $\mathrm{Si}$ material) and is transparent at the standard fiber optical communication wavelengths $(1.3-1.6 \mu \mathrm{m})$ [3]. Silicon exhibit optical properties that are useful for a variety of applications [5]. Its processing has been developed by the electronics industry to a level that is more than sufficient for most integrated optical applications [4]. Due to the high refractive index of silicon ( 3.5 approx.), one can make significantly smaller photonic devices as compared to those made in a low index medium (e.g. silica $n=1.45$ ) since the high index contrast. Also, its large refractive index allows for a tight confinement of optical waves to a sub-micron region using the SOI technology [3]. SOI wafers, with large sizes and high quality, are commercial available and an ideal platform for creating planar waveguides $[1,2,5]$. Because the strong optical confinement offered by the high index contrast between silicon and $\mathrm{SiO}_{2}$, SOI waveguides can exhibit a large third-order nonlinearity, with Kerr coefficient more than 100 times larger than those of silica glass in the telecommunication band [6]. However, it is important to remember that silicon is a semiconductor crystal exhibiting unique features such TPA, FCD, FCA, anisotropic and dispersive third-order nonlinearity [7]. The interplay among various optical properties leads to many interesting features that provide new functionalities on the one hand, but may become obstacles in some cases on the other [7]. 


\subsection{SOI Waveguides Characteristics}

There is no doubt that guided wave optical devices at nanoscale will have an increasing impact on integrated optics in the coming years. Primarily, this is because of the sustained development of two extremely successful components: SOI waveguides and the semiconductor laser. Together, these are currently in the progress of revolutionizing the optical interconnects on the chip level, and making considerable advances in a variety of other new and exciting application areas. For example, the all-optical switch based on coupled silicon nanowires may be used to perform logic operations for the optical signal processing on high-speed optical on-chip networks.

Theoretical and experimental studies have been made of optical waveguides produced using the SOI technology (see for example Q. Lin, et al. [7] and J. Zhang, et al. [8]). Soliton formation and propagation in short SOI waveguides have been reported by some important research groups [8]. Since SOI waveguide acts as a propagation medium for guided optical waves, and is the first essential component in silicon photonic technology [3], therefore, it is essential to appreciate the structure of SOI waveguides in order to understand the difficulties associated with some aspects of optical circuit design in integrated optics. SOI waveguides, widely used in photonic research, consists of a three-layer structure comprising a core and two cladding layers. The core is usually all silicon ( $\mathrm{Si}$ ) with a constant refractive index. It is typically of the order of hundreds of nanometers in thickness. The cladding consists of buried silicon dioxide (buried $\mathrm{SiO}_{2}$ ) and it is typically about half a micron, although individual designs vary [4]. The purpose of the buried oxide layer is to act as the lower cladding layer, and hence prevents the field associated with the optical modes from penetrating the silicon substrate below [3-4]. Four novel waveguide configurations are demonstrated 
[9]. In all of these $\mathrm{SiO}_{2}$ is used as an optical buffer between the guiding layer and the silicon substrate. The most standard waveguides used for silicon photonics are channel waveguides and ridge waveguides (see Fig. 1 [9]). The operating wavelengths for these waveguides is in the vicinity of the $1.55 \mu \mathrm{m}$ infrared band (wavelength that corresponds to the maximum transparency of the silica fiber), suitable for optical communication.

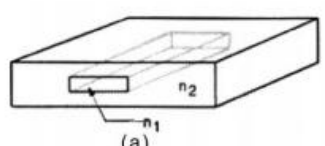

$(\mathrm{a})^{n_{1}}$

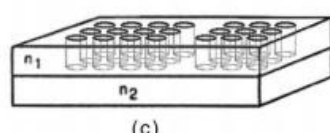

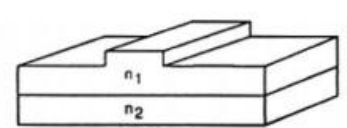

(b)

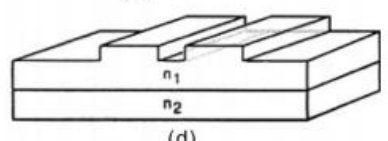

(d)

Figure 1. Four configurations of waveguides in silicon: (a) Chanel waveguides; (b) Rib waveguides; (c) Photonic-crystal waveguides; (d) Slot waveguides. (Source: [9]).

SIO waveguides with nanoscale dimensions are commonly referred as photonic nanowires, photonic wires or just silicon nanowires. They allow for small size waveguides in the sub-micrometer range, and therefore a large number of optical components like power splitters and combiners, optical switches and modulators, optical filters and attenuators, wavelength division multiplexers to be integrated within a small area $[3,10]$. These wave guiding devices play important roles in applications from optical fiber telecommunications to chip-to-chip optical interconnection. Among them, optical switches and modulators are core components for dense wavelength division multiplexing (DWDM), optical cross connection (OXC) and optical add/drop multiplexing (OADM) [10]. Silicon nanowires have high propagation losses (due to scattering off the waveguide's sidewalls) and high fiber-towaveguide coupling losses $[8,11]$. However, it has been demonstrated that 
single-mode photonic wires, in silicon-on-insulator, reach propagation losses as low as $2.4 \mathrm{~dB} / \mathrm{cm}[11]$.

Since we are interested on the analysis and application of the nonlinear directional coupler with micrometer dimensions and fabricated with the SOI technology; single-mode rib waveguides with small cross-section dimensions, which allows two-dimensional confinement i.e. about $0.1 \mu \mathrm{m}^{2}$, which allows two-dimensional confinement, are the material of this work. In ridge waveguides, the guiding layer consists of a slab with a dielectric ridge on top embedded between two low-index-of-refraction layers. In both of them, the channel and the ridge waveguide, the light is confined due to the total internal reflection between the high- and low-index-of-refraction regions [9].

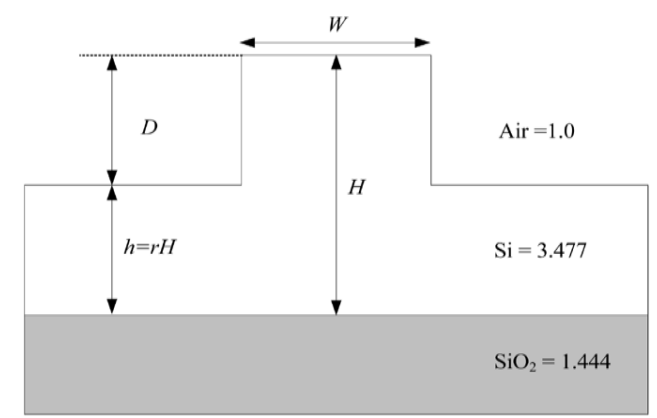

Figure 2. Cross section and waveguide parameters of an SOI rib waveguide. (Source: [13]).

The most important SOI waveguide type is the single-mode silicon waveguide which can support only one mode. Single-mode operation is highly required for most optical waveguide devices [12]. In order to design devices accurately, it is necessary to determine the single mode condition (SMC) of the silicon waveguides. The fabrication restrictions for small SOI rib waveguides, with deeply etched ribs ( $1 \mu \mathrm{m}$ approx. or less in cross section), must be imposed on their geometry. To make them behave as single-mode devices, and at the same time polarization-independent, they must satisfy a generalized relation in terms of waveguide dimensions [13] 


$$
\frac{W}{H} \leq 0.05+\frac{(0.94+0.25 H) r}{\sqrt{1-r^{2}}}
$$

where $0.3<r<0.5$ and $1.0 \leq \mathrm{H} \leq 1.5, \mathrm{r}$ is the ratio of slab height to overall rib height and $\mathrm{W} / \mathrm{H}$ is the ratio of waveguide width to overall rib height.

\subsection{Objective}

The objective of this thesis is to obtain a theoretical platform for the design of a silicon waveguide integrated optical switch based on coupled silicon nanowires, with high switching speed. We wish to obtain a theoretical analysis of the coupled system, with micrometer dimensions and made with the SOI technology, by using a rigorous full-vectorial method. There, we will consider the vector nature of the optical modes and the effects of the twophoton absorption on various nonlinear processes. Based on the analytical treatment of the nonlinear directional coupler, we wish for the physical insight that is often lost in numerical simulations. We intent to derive the analytical solutions for the coupled-mode equations in some cases in which the density of the free carrier is relatively low, and dispersive effects are relatively weak, assuming that the nonlinear effects do not affect the waveguide modes significantly. Although the switching characteristics can be altered for a free carrier based device at the expense of extra excess loss, a switch behavior of the coupled system is pursued.

\subsection{Synopsis}

In general, this work is directed to describe the nonlinear effects in semiconductor waveguides for optical switching and two particular SOI 
waveguides are studied. In the first chapter, we give a short introduction based on three lines: the context of this work, silicon photonics and SOI waveguides. In the second chapter, we review some important literature about the novel characteristics of SOI waveguides. And we give an overview of phenomena in silicon waveguides that are important in the characterization of photonic devices. In the third chapter, we develop a model of the nonlinear propagation of optical pulses through coupled silicon, taking into account a wide range of physical effects. This includes the third-order nonlinear effect, TPA, FCA, FCD, the linear and nonlinear losses and the coupling effect. We obtain the general coupled-mode equations for the nonlinear directional coupler with micrometer dimensions. In the fourth chapter, we introduce a few particular analytical solutions for the coupledmode equations in those cases in which the free carrier density is relatively low, and dispersive effects are relatively weak, assuming that the nonlinear effects do not affect the waveguide modes significantly and using physical parameters based upon experimental investigation. Finally, conclusions are given in chapter 5 .

\section{References}

[1] R. A. Soref, "The past, Present, and Future of Silicon Photonics," IEEE J. Sel. Top. Quantum Electron. 12, 1678-1687 (2006).

[2] R. A. Soref, "The achievements and Challenges of Silicon Photonics", invited lead article in Advances in Optical Technology (Hindawi, online), special issue on silicon photonics (2008).

[3] L. Pavesi and D. J. Lockwood, Eds., Silicon Photonics (Springer, New York, 2004). 
[4] G. T. Reed and A. P. Knights, Silicon Photonics: An introduction (Wiley, Hoboken, NJ, 2004).

[5] B. Jalali and S. Fathpour, "Silicon Photonics," J. Lightwave Technol. 24, 4600-4615 (2006).

[6] M. Dinu, F. Quochi, and H. Garcia, "Third-order nonlinearities in silicon at telecom wavelengths," Appl. Phys. Lett. 82, 2954-2956 (2003).

[7] Q. Lin, O. J. Painter, and G. P. Agrawal, "Nonlinear optical phenomena in silicon waveguides: modeling and applications," Opt. Express 15, 1660416644 (2007).

[8] J. Zhang, Q. Lin, G. Piredda, R. W. Boyd, G. P. Agrawal, and P. M. Fauchet, "Optical solitons in a silicon waveguide," Opt. Express 15, 76827688 (2007).

[9] M. Lipson, "Guiding, modulating, and emitting light on silicon - challenges and opportunities," J. Lightwave Technol. 23, $4222-4238$ (2005).

[10] R. Soref, "Recent Advances in Silicon Photonic Components," in Integrated Photonics Research and Applications/Nanophotonics for Information Systems, Technical Digest (Optical Society of America, 2005), paper JWA1.

[11] W. Bogaerts et al, Opt. Lett. 28(9), p. 689

[12] J. Yu, S. Chen, Z. Li, Y. Chen, F. Sun, Y. Li, Y. Li, J. Liu, D. Yang, J. Xia, C. Li and Q. Wang, "Recent progress of SOI-based photonic devices", Proc. SPIE 6020, 60201R (2005); doi:10.1117/12.633878

[13] Seong Phun Chan, Ching Eng Png, Soon Thor Lim, Graham T. Reed, and Vittorio M. N. Passaro, "Single-Mode and Polarization-Independent Silicon-on-Insulator Waveguides With Small Cross Section," J. Lightwave Technol. 23, 2103- (2005). 


\section{Chapter 2}

\section{Novel Properties of SOI Waveguides}

This chapter is intended to introduce some important properties of the SOI waveguides, before we embark into a full theoretical analysis of the nonlinear propagation of optical pulses through silicon waveguides at the nanoscale dimensions. First, we will consider the dispersion in silicon waveguides and show that the SOI waveguide dimensions determine the group-velocity dispersion (GVD) [1,2]. Then, we introduce the material dispersion briefly, and finally, we show the dependence of relevant nonlinear parameters on the waveguide dimensions.

\subsection{Dispersion in SOI Waveguides}

It can be shown that waveguide geometry dominates the dispersive properties of SOI waveguides [1-3]. The GVD in photonic nanowires can be highly-controlled, while remaining close to the optimal effective nonlinearity [1], due to the high confinement sensitivity to wavelength and to waveguide dimensions. Since the zero-dispersion wavelength (ZDWL) of a SOI waveguide can be shifted around $1.55 \mu \mathrm{m}$ with reasonable device parameters, silicon waveguides can exhibit anomalous dispersion $\left(\beta_{2}<0\right)$ [2]. In the nonlinear regime, the combination of dispersion and nonlinearity 
result in the formation of solitons. They contain a range of frequency components, and are bound together by the balance between the dispersion of these frequency components and the nonlinearity of the system. This characteristic may lead to new applications of SOI waveguides related to optical interconnects and high-speed optical switching [2].

\subsubsection{Material dispersion of silicon}

The refractive index of any medium is a function of wavelength, and hence different wavelengths that see different refractive indices will

propagate with different velocities, resulting in material dispersion (also named chromatic dispersion). This type of dispersion results from the fact that any optical source is not purely monochromatic, so the different spectral components of the light source may have different propagation delays, and hence pulse broadening may occur, even if transmitted by a single mode. The material dispersion of silicon is well approximated by the Sellmeier relation [3]

$$
\mathrm{n}(\lambda)=\varepsilon+\frac{\mathrm{A}}{\lambda^{2}}+\frac{\mathrm{B} \lambda_{1}^{2}}{\lambda^{2}-\lambda_{1}^{2}}
$$

where $\quad A=0.939816 \mu \mathrm{m}^{2}, \quad B=8.10461 \times 10^{-3}, \quad \lambda_{1}=1.1071 \mu \mathrm{m} \quad$ and $\varepsilon=11.6858$. This relation is agreeing with experimental data [3]. In the range of interest (around $1.55 \mu \mathrm{m}$ ), the system is far from of the resonance and there is not absorption of electromagnetic radiation. Since the birefringence of silicon corresponds to a refractive index difference of less than $10^{-5}$, the refraction index of silicon can be approximated to a scalar quantity [4]. 


\subsubsection{Engineered dispersion}

Mathematically, the effects of waveguide dispersion are accounted for by expanding the mode-propagation constant in a Taylor series about the frequency $\omega_{0}$ at which the pulse spectrum is centered:

$$
\beta(\omega)=n(\omega) \frac{\omega}{c}=\beta_{0}+\beta_{1}\left(\omega-\omega_{0}\right)+\frac{1}{2} \beta_{2}\left(\omega-\omega_{0}\right)^{2}+\cdots
$$

where the dispersion coefficient of the mth order is defined as

$$
\beta_{\mathrm{m}}=\left(\frac{\mathrm{d}^{\mathrm{m}} \beta}{\mathrm{d} \omega^{\mathrm{m}}}\right)_{\omega=\omega_{0}}(\mathrm{~m}=0,1,2, \ldots) .
$$

The parameters $\beta_{1}$ and $\beta_{2}$ are related to the group velocity $v_{g}$ through the relations

$$
\begin{aligned}
& \beta_{1}=\frac{1}{\mathrm{v}_{\mathrm{g}}}=\frac{\mathrm{n}_{\mathrm{g}}}{\mathrm{c}} \\
& \beta_{2}=\frac{\mathrm{d}}{\mathrm{d} \omega}\left(\frac{1}{\mathrm{v}_{\mathrm{g}}}\right)
\end{aligned}
$$

where $n_{g}$ is the group index and $v_{g}$ is the group velocity [5]. Physically speaking, the envelope of an optical pulse moves at the group velocity while the parameter $\beta_{2}$ represents dispersion of the group velocity and is responsible for pulse broadening. This phenomenon is known as the GVD, and $\beta_{2}$ is the GVD parameter [5]. The GVD is normal or anomalous depending on whether $\beta_{2}$ is positive or negative. The GVD controls the broadening of ultrafast pulses and in a waveguide geometry is determined by 
both the intrinsic material dispersion and by a contribution from the confinement of the waveguide [1].

The dispersive properties of SOI waveguides have been studied in [2] by using the effective-index method. Results indicated that based on reasonable device parameters, an ultra-short pulse at $1.55 \mu \mathrm{m}$ should form a soliton as it propagates in the waveguide. The wavelength dependence of the secondorder dispersion parameter for the TE and TM waveguide modes is shown in Fig. 3.

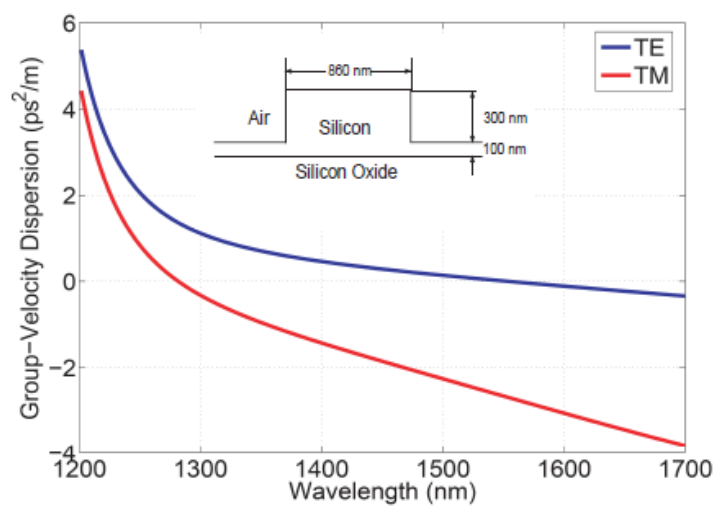

Figure 3. Calculated dispersion curves for the TE and TM modes. The design of their waveguide is shown in the inset (Source: [6]).

This numerical calculation was made using waveguides with dimensions of $860 \times 400 \mathrm{~nm}^{2}$ in the cross section, and an etching depth of $300 \mathrm{~nm}$. These waveguide parameters will be used for our physical system. The fundamental TM mode exhibits a GVD of $-2.26 \mathrm{ps}^{2} / \mathrm{m}$ at $1500 \mathrm{~nm}$. This value is more than 100 times larger than that of standard silica fibers $\left(<0.02 \mathrm{ps}^{2} / \mathrm{m}\right)[6]$.

\subsection{Geometric Dependence of Nonlinear Parameters}

Some investigations done in the field of silicon nanowires show that the effective nonlinear parameters that govern the pulse propagation in silicon 
nanowires depend on waveguide dimensions (see for example Daniel and Agrawal [7]).

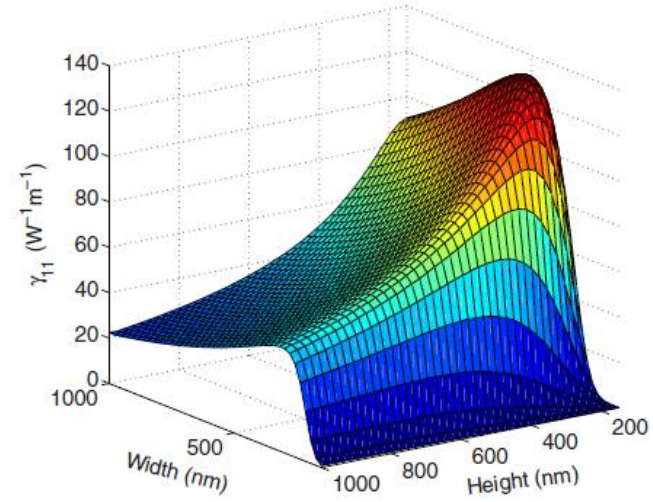

Figure 4. Nonlinear parameter $\gamma_{11}$ as a function of waveguide width and height at the 1550 nm wavelength (Source: [7]).

The geometric dependence of these quantities was investigated in [7] using channel waveguides whose rectangular silicon core is surrounded on all sides by silica. Their results indicated that the nonlinear parameter of a mode depends strongly on the dimension of the waveguide that is parallel to the mode's polarization direction. Anisotropy of silicon plays a important role in these results. Figure 4, shows the geometrical dependence of the nonlinear parameters.

\subsection{Summary}

In summary, we inspected the dispersive properties of SOI waveguides and checked the wavelength dependence of the GVD parameter $\beta_{2}$ for TE and TM waveguides modes. This calculations was made in [7] for the waveguide dimension that will be used in this work. We revised as silicon waveguides present the effect of dispersion tailoring which causes that the dispersion relation can be modified by altering the geometry of the 
waveguide. Also, we revised the dependence of relevant nonlinear parameters on waveguide dimensions.

\section{References}

[1] Mark A. Foster, Amy C. Turner, Michal Lipson, and Alexander L. Gaeta, "Nonlinear optics in photonic nanowires," Opt. Express 16, 1300-1320 (2008).

[2] L. Yin, Q. Lin, and G. P. Agrawal, "Dispersion tailoring and soliton propagation in silicon waveguides," Opt. Lett. 31, 1295-1297 (2006).

[3] R. M. Osgood, Jr., N. C. Panoiu, J. I. Dadap, X. Liu, X. Chen, I. W. Hsieh, E. Dulkeith, W. M. Green, and Y. A. Vlasov, "Engineering nonlinearities in nanoscale optical systems: physics and applications in dispersionengineered silicon nanophotonic wires," Adv. Opt. Photon. 1, 162-235 (2009).

[4] D. Kovalev, G. Polisski, J. Diener, H. Heckler, N. Knzner, V. Yu. Timoshenko, and F. Koch. Strong in-plane birefringence of spatially nanostructured silicon. Appl. Phys. Lett. 78:916, 2001.

[5] G. P. Agrawal, Nonlinear Fiber Optics (Academic, 2007).

[6] J. Zhang, Q. Lin, G. Piredda, R. W. Boyd, G. P. Agrawal, and P. M. Fauchet, "Observation of optical solitons in a silicon waveguide," Opt. Exp. 15, 7682-7688 (2007).

[7] B. A. Daniel and G. P. Agrawal, "Vectorial nonlinear pulse propagation in silicon nanowire waveguides: polarization effects," J. Opt. Soc. Am. B 27, 956-965 (2010). 


\section{Chapter 3}

\section{Theoretical Framework}

In this chapter we set the fundamental equations for a complete description of nonlinear propagation in coupled semiconductor waveguides. Principally instantaneous nonlinear processes and free-carrier effects are theoretically investigated and included in the modeling for an electromagnetic analysis of the evolution of an optical pulse in an array of $M$ silicon nanowires made with the SOI technology. The material presented here is deduced for the first time, although it is based in some investigations already done in the literature (see for example Daniel and Agrawal [1]).

\subsection{The Wave Equation for Silicon}

Consider the rational theory for the propagation of light that was developed by Maxwell in the 1860s. The equations for the electric and magnetic intensity fields $\mathbf{E}(\mathbf{r}, \mathrm{t})$ and $\mathbf{H}(\mathbf{r}, \mathrm{t})$ in MKS units are given by

$$
\begin{gathered}
\nabla \times \mathbf{E}=-\mu_{0} \frac{\partial \mathbf{H}}{\partial \mathrm{t}} \\
\nabla \times \mathbf{H}=\sigma \mathbf{E}+\varepsilon_{0} \frac{\partial \mathbf{E}}{\partial \mathrm{t}}+\frac{\partial \mathbf{P}}{\partial \mathrm{t}},
\end{gathered}
$$


where $\mu_{0}$ is the magnetic constant, $\varepsilon_{0}$ is the electric constant, $\sigma$ is the conductivity, and $\mathbf{P}(\mathbf{r}, \mathrm{t})$ is the polarization density. We have ignored the effect of magnetization, which is justified, as silicon's relative permeability differs from unity by less than $10^{-5}$. The general second-order wave equation for the propagation of an optical field in a silicon like optical medium, valid for both linear and nonlinear characterization, can be obtained by taking the curl of Eq. (3.1) and using Eq. (3.2), that is

$$
-\nabla(\nabla \cdot \mathbf{E})+\nabla^{2} \mathbf{E}=\frac{1}{\mathrm{c}^{2}} \frac{\partial^{2} \mathbf{E}}{\partial \mathrm{t}^{2}}+\mu_{0} \frac{\partial^{2} \mathbf{P}}{\partial \mathrm{t}^{2}},
$$

where $c$ is the speed of light in vacuum and we have used the relation $\mu_{0} \varepsilon_{0}=1 / \mathrm{c}^{2}$. In the above equation we have applied the vector identity $\nabla \times(\nabla \times \mathbf{E})=\nabla(\nabla \cdot \mathbf{E})-\nabla^{2} \mathbf{E}$. The solution of the wave equation for waveguides mathematically describes the optical modes of the waveguides and how one field waveguide relates to another in an optical coupling process from one waveguide to another. Let's notice, that from the start and for convenience to ease the mathematics, we have assumed the simplification that the waveguide material is non-conducting (i.e. $\sigma=0$ ). However, theoretically we will introduce the free-carrier effects in the wave equation for a general modeling of the phenomena in the semiconductor material.

The nature of silicon is exhibited in the relation between $\mathbf{P}$ and $\mathbf{E}$, called the medium equation. In general, silicon is nonlinear, dispersive and anisotropic media [2-4]. On a fundamental level, the origin of nonlinear response is related to anharmonic motion of bound electrons under the influence of an applied field. As a result, the total polarization $\mathbf{P}$ induced by electric dipoles is not linear in the electric field $\mathbf{E}$, but satisfies the more general relation [5] 


$$
\mathbf{P}=\varepsilon_{0}\left(\chi^{(1)} \cdot \mathbf{E}+\chi^{(2)}: \mathrm{EE}+\chi^{(3)}: \mathrm{EEE}+\cdots\right),
$$

where $\chi^{(j)}(j=1,2, \ldots)$ is the $j$ th order susceptibility. In general, $\chi^{(j)}$ is a tensor of rank $j+1$. We are considering optical nonlinearities with more mathematical rigor as usual because most optically nonlinear materials, as silicon, are anisotropic and demand the use of tensors to describe their linear and nonlinear properties [4]. It is also convenient to split $\mathbf{P}$ into its linear and nonlinear parts as $\mathbf{P}=\mathbf{P}^{(1)}+\mathbf{P}^{\mathrm{NL}}$, where $\mathbf{P}^{(1)}$ is the part of polarization that depends linearly on the electric field $\mathbf{E}$ and $\mathbf{P}^{\mathrm{NL}}$ is the nonlinear polarization. Introducing $\mathbf{E}$ in terms of its Fourier transform through the relation

$$
\mathbf{E}(\mathbf{r}, \mathrm{t})=\frac{1}{2 \pi} \int_{-\infty}^{\infty} \tilde{\mathbf{E}}(\mathbf{r}, \omega) \mathrm{e}^{-i \omega t} \mathrm{~d} \omega
$$

as well as similar relation for $\mathbf{P}$ in Eq. (3.3), we obtain a vector wave equation in Fourier space for monochromatic fields given by

$$
-\nabla(\nabla \cdot \tilde{\mathbf{E}})+\nabla_{\mathrm{T}}^{2} \tilde{\mathbf{E}}+\frac{\partial^{2} \tilde{\mathbf{E}}}{\partial \mathbf{z}^{2}}+\frac{\omega^{2}}{\mathrm{c}^{2}} \mathrm{n}_{0}^{2}(\mathbf{r}, \omega) \tilde{\mathbf{E}}+\omega^{2} \mu_{0} \tilde{\mathbf{P}}^{N L}=0,
$$

where $\omega$ is the angular frequency and the induced polarization of first order is given by $\tilde{\mathbf{P}}^{(1)}(\mathbf{r}, \omega)=\varepsilon_{0} \chi^{(1)} \cdot \tilde{\mathbf{E}}(\mathbf{r}, \omega)$. In general, $\chi^{(1)}$ is assumed to be a scalar and related with the refractive index $n_{0}$ of the whole system through $\mathrm{n}_{0}^{2}=1+\chi^{(1)}$. The corresponding Laplacian is given by $\nabla^{2}=\partial^{2} / \partial \mathrm{z}^{2}+\nabla_{\mathrm{T}}^{2}$.

For the nonlinear polarization $\tilde{\mathbf{P}}^{\mathrm{NL}}$ in Eq. (3.6), since $\chi^{(2)}=0$ for silicon $[2,4,6]$, we only consider the third-order Kerr nonlinearity for which we can approximate the nonlinear polarization by $\tilde{\mathbf{P}}^{\mathrm{NL}} \approx \tilde{\mathbf{P}}^{(3)}$, where $\tilde{\mathbf{P}}^{(3)}=\varepsilon_{0} \chi^{(3)}: \tilde{\mathbf{E} E \tilde{E}} \tilde{E}$ and the higher order nonlinear effects are negligible. However, it is important to remember that silicon is a semiconductor crystal exhibiting unique features 
such as TPA, which contributes to the material nonlinear polarization $\tilde{\mathbf{P}}^{\mathrm{NL}}$. In semiconductor waveguides, free-carriers generated by TPA could lead to additional losses through FCA and refractive index changes through FCD. Therefore, it is important to include the free-carrier effects in the analysis of optical pulses propagating through silicon waveguides. After including the induced polarizations associated with the third-order nonlinearity and freecarriers generated by TPA, the wave equation in the frequency domain Eq. (3.6) takes the form [2]

$$
-\nabla(\nabla \cdot \tilde{\mathbf{E}})+\nabla_{\mathrm{T}}^{2} \tilde{\mathbf{E}}+\frac{\partial^{2} \tilde{\mathbf{E}}}{\partial \mathbf{z}^{2}}+\frac{\omega^{2}}{\mathrm{c}^{2}} \mathrm{n}_{0}^{2}(\mathbf{r}, \omega) \tilde{\mathbf{E}}=-\omega^{2} \mu_{0}\left[\tilde{\mathbf{P}}^{f}(\mathbf{r}, \omega)+\tilde{\mathbf{P}}^{(3)}(\mathbf{r}, \omega)\right] .
$$

In general, the induced polarizations are only small perturbations of the linear wave equation. Equation (3.7) is the most general wave equation for silicon, and describes the propagation of electromagnetic waves in it. The solution of the nonlinear wave equation for silicon waveguides mathematically describes the waveguides optical modes.

\subsection{Coupled-Mode Equations}

Thus far we have essentially built the vector wave equation for silicon. This section is included to learn how the nonlinear optical wave equation, that we derived in the previous section, can be used to obtain the coupled-mode equations that describe the behavior of light inside an array of silicon waveguides. For this, we assume that the array consists of $M$ identical singlemode nanowires. From the coupled mode theory [7], we know that the guide, or bound, power along each nanowire in the array can be expressed as a finite sum of bound modes of every silicon nanowire. All the individual modes of the set are the source-free solutions to Maxwell's equations for 
each nanowire and do not satisfy the Maxwell's equations of the whole system.

The longitudinal evolution of the electromagnetic field inside each waveguide during the propagation can be described by a z-dependent amplitude $\tilde{a}$ to be found through coupling equations. The z-dependent amplitude of each mode is determined by a set of coupled-mode equations that are obtained using the well-known coupled-mode approach [7]. This approximation is based on the orthogonal modal fields that have unit normalization. Vectorial solutions for Eq. (3.7) results in a complete and finite orthogonal set of forward, backward and radiation propagation modes; which are the source-free solutions to Maxwell's equations for the waveguide. However, in this work we only consider forward-propagating modes which travel in the positive z-direction, for which the solution of Eq. (3.7) can be expressed by

$$
\tilde{\mathbf{E}}(\mathbf{r}, \omega) \approx \sum_{\mathrm{m}=1}^{\mathrm{M}} \tilde{a}_{\mathrm{m}}\left(\mathrm{z}, \omega-\omega_{0}\right) \frac{\mathbf{e}_{\mathrm{m}}\left(\mathrm{x}, \mathrm{y}, \omega_{0}\right)}{\sqrt{\mathrm{N}_{\mathrm{m}}}} \mathrm{e}^{\mathrm{i} \beta(\omega) \mathrm{z}},
$$

where $\mathbf{e}_{\mathrm{m}}$ is the mode structure of the mth nanowire in the absence of other nanowires, $\tilde{a}_{\mathrm{m}}$ is the slowly varying modal amplitude in the frequency domain of the mth waveguide, $\beta$ is the total propagation constant of the field inside the array, $\omega_{0}$ is the carrier frequency, and the superscript $m$ is an integer number between 1 to $M$; where $M$ is the total number of waveguides in the array. Let's notice that Eq. (3.8) is a superposition of the evolution of the optical modal fields of the single nanowires which expresses the total field evolution through the array of silicon waveguides. Also, in the previous equation, we approximated the frequency dependence of the fields to be totally contained within the modal coefficients $\tilde{a}_{m}$ and we have normalized 
the transversal profile of the electromagnetic field where the parameter $\mathrm{N}_{\mathrm{m}}$ represents the transversal distribution of power flows parallel to the $\mathrm{mth}$ waveguide axis which is defined as [7]

$$
\mathrm{N}_{\mathrm{m}}=\frac{1}{2} \iint\left(\mathbf{e}_{\mathrm{m}} \times \mathbf{h}_{\mathrm{m}}^{*}\right) \cdot \hat{\mathbf{z}} \mathrm{dxdy},
$$

where the integrals extend over the core cross-section. The asterisk denotes the complex conjugation and $\hat{\mathbf{z}}$ is the unit vector parallel to the waveguide axis. The above relation expresses the combination of the vector orthogonality condition and the normalization for one forward-propagating mode.

For convenience throughout this work, we assume that the nonlinear effects do not affect the mode significantly. Therefore, the fundamental mode supported by the mth nanowire in the absence of other nanowires can be represented by $\tilde{\mathbf{E}}^{(\mathrm{m})}(\mathbf{r}, \omega)=\mathbf{e}_{\mathrm{m}}(\mathrm{x}, \mathrm{y}, \omega) \mathrm{e}^{\mathrm{i} \beta_{\mathrm{m}}(\omega) \mathrm{z}}$, where $\beta_{\mathrm{m}}$ is the propagation constant corresponding to the $\mathrm{mth}$ nanowire. One necessary condition for the fundamental modes (i.e. $\tilde{\mathbf{E}}^{(m)}$ ) is that the fields fall off exponentially outside the silicon cores of the array of silicon nanowires because they are even functions.

The basic mode solutions are obtained by solving Eq. (3.7) and satisfy the following equation:

$$
-\nabla\left(\nabla \cdot \tilde{\mathbf{E}}^{(\mathrm{m})}\right)+\nabla_{\mathrm{T}}^{2} \tilde{\mathbf{E}}^{(\mathrm{m})}+\frac{\partial^{2} \tilde{\mathbf{E}}^{(\mathrm{m})}}{\partial \mathbf{z}^{2}}+\frac{\omega^{2}}{\mathrm{c}^{2}} \mathrm{n}_{0 \mathrm{~m}}^{2}(\mathbf{r}, \omega) \tilde{\mathbf{E}}^{(\mathrm{m})}=0,
$$

where $\mathrm{n}_{0 \mathrm{~m}}$ is the linear refraction index function corresponding to the mth waveguide, in the absence of other nanowires. Note that Eq. (3.10) governs the transverse behavior of the electric field. The vectorial function $\mathbf{e}_{\mathrm{m}}$ must satisfy the continuity conditions at the interfaces of the waveguides. 
Substituting Eq. (3.8) into Eq. (3.7), applying the slowly varying envelope approximation and assuming that the light is spectrally narrow, we obtain after associating terms (see the Appendix A for its derivation)

$$
\frac{\partial \tilde{a}_{\mathrm{k}}}{\partial \mathrm{z}}=\mathrm{i}\left(\beta_{\mathrm{k}}-\beta\right) \tilde{a}_{\mathrm{k}}+\mathrm{i} \sum_{\mathrm{m}=1}^{\mathrm{M}} \kappa_{\mathrm{km}} \tilde{a}_{\mathrm{m}}+\frac{\mathrm{i} \omega \mathrm{e}^{-i \beta z}}{4 \sqrt{\mathrm{N}_{\mathrm{k}}}} \iint \mathbf{e}_{\mathrm{k}}^{*} \cdot\left(\tilde{\mathbf{P}}^{\mathrm{f}}+\tilde{\mathbf{P}}^{(3)}\right) \mathrm{dxdy} .
$$

The terms shown on the right hand of this equation correspond respectively to the dispersion, coupling and nonlinearity. The integrals extend over the entire $x-y$ plane and no perturbation has been considered for the dispersion and nonlinearity. This result represents the more general relation that the mode amplitudes satisfy. Here, the parameter $\kappa_{\mathrm{km}}$ is the coupling coefficient and is given by

$$
\kappa_{\mathrm{km}}=\frac{\omega \varepsilon_{0}}{4 \sqrt{N_{\mathrm{k}} \mathrm{N}_{\mathrm{m}}}} \iint \mathbf{e}_{\mathrm{k}}^{*} \Delta \mathrm{n}_{\mathrm{m}}^{2} \mathbf{e}_{\mathrm{m}} \mathrm{dxdy}
$$

where $\Delta n_{m}^{2}=n_{0}^{2}-n_{0 m}^{2}$ is the change in the refraction index due to the presence of the mth guide mode.

We are interested to rewrite Eq. (3.11) into the time domain. For this purpose we can replace the propagation constant $\beta_{k}$ with its Taylor expansion around $\omega_{0}$ as:

$$
\beta_{k}(\omega)=\sum_{n=0}^{\infty} \mathrm{i}^{\mathrm{n}} \frac{\beta_{\mathrm{kn}}}{\mathrm{n} !}\left(\omega-\omega_{0}\right)^{\mathrm{n}}
$$

where $\beta_{k n}$ are the dispersion coefficients defined as $\beta_{k n}=\left.\left(d^{n} \beta_{k} / d \omega^{n}\right)\right|_{\omega=\omega_{0}}$. The constant $\beta_{\mathrm{k} 0}$, as well as the first-order parameter $\beta_{\mathrm{k} 1}$ and the coefficient $\beta_{\mathrm{k} 2}$ are important phase-factors that have a significant weight in our mathematical model. Note that the core frequency $\omega_{0}$ is chosen to be the pump frequency. This is the principal frequency of the light fired into the 
nanowire. Therefore, replacing $\omega-\omega_{0}$ with $\mathrm{i} \partial / \partial t$ and multiplying both sides of Eq. (3.11) by $e^{-i \omega t}$ and integrate with respect to $\omega$, we obtain

$$
\begin{aligned}
\frac{\partial a_{\mathrm{k}}}{\partial \mathrm{z}}=\left[\sum_{\mathrm{n}=1}^{\infty} \mathrm{i}^{\mathrm{n}+1} \frac{\beta_{\mathrm{kn}}}{\mathrm{n} !} \frac{\partial^{\mathrm{n}}}{\partial \mathrm{t}^{\mathrm{n}}}\right] a_{\mathrm{k}}+\mathrm{i} \sum_{\mathrm{m}=1}^{\mathrm{M}} \kappa_{\mathrm{km}} a_{\mathrm{m}}+\mathrm{i} \delta_{\mathrm{km}} a_{\mathrm{k}} \\
+\frac{\mathrm{i} \omega_{0} \mathrm{e}^{-\mathrm{i}\left(\left(\beta z-\omega_{0} \mathrm{t}\right)\right.}}{4 \sqrt{\mathrm{N}_{\mathrm{k}}}} \iint \mathbf{e}_{\mathrm{k}}^{*} \cdot\left(\mathbf{P}^{\mathrm{f}}+\mathbf{P}^{(3)}\right) \mathrm{dxdy} .
\end{aligned}
$$

where $\delta_{\mathrm{km}}$ is the asymmetry parameter given by

$$
\delta_{\mathrm{km}}=\frac{(\mathrm{m}-1) \beta_{\mathrm{k} 0}}{\mathrm{~m}}-\sum_{\mathrm{j} \neq \mathrm{k}} \frac{\beta_{\mathrm{j} 0}}{\mathrm{~m}} .
$$

The TPA phenomena may generate a significant number of free electrons and holes as a function of the peak power associated with the incident optical field. The contribution of free-carriers to the nonlinear polarization is given by [1-2]

$$
\mathbf{P}^{\mathrm{f}}(\mathbf{r}, \mathrm{t})=2 \mathrm{n}_{0} \varepsilon_{0}\left[\Delta \mathrm{n}^{\mathrm{f}}+\left(\mathrm{i} / 2 \mathrm{k}_{0}\right) \Delta \alpha^{\mathrm{f}}\right] \mathbf{E}(\mathbf{r}, \mathrm{t}),
$$

where $k_{0}=\omega_{0} / c$ and $n_{0}$ is the refractive index of bulk silicon. The Freecarrier index (FCI) change and FCA are described by the quantities $\Delta \mathrm{n}^{f}$ and $\Delta \alpha^{f}$, both of which implicitly depend on the excess carrier density. The response of the free-carrier induced polarization is extremely fast, as in the nonlinear response, and can be taken as instantaneous [4]. From the theory of the dynamics of free carriers we found that the free-carrier induced polarization varies linearly with the densities of free electrons and holes, $\mathrm{N}_{e}$ and $N_{h}$, respectively [2]. To simplify, we assume equal carrier concentrations and lifetimes for electrons and holes, i.e., $N_{e}=N_{h}=N$. In this case we can use the following approximation for the FCI and FCA [2] 


$$
\Delta \alpha^{f}=\sigma_{a}(\omega) \mathrm{N}, \quad \Delta \mathrm{n}^{\mathrm{f}}=-\sigma_{\mathrm{n}}(\omega) \mathrm{N},
$$

where $\sigma_{a}$ is the free-carrier absorption cross section and $\sigma_{\mathrm{n}}$ is the freecarrier dispersion coefficient.

In our model of interaction of radiation with matter, we have assumed that a dipole can be created by an electric field and that the restoring force on the charges is proportional to the displacement. This led to a form of simple harmonic motion, and to a polarization proportional to the driving field. In Section 3.1 we have expressed the form of this general polarization, however, at this moment we are only interested in the third-order polarization which characterizes the nonlinear response of silicon. Therefore the electronic response, that is due to electrons bound to silicon atoms in the crystal lattice, is very fast and can be taken as instantaneous $[1-2,4,6]$. We can write the third-order polarization in the following form [11]

$$
\mathbf{P}^{(3)}(\mathbf{r}, \mathrm{t})=\frac{3}{4} \varepsilon_{0} \chi^{(3)}\left(-\omega_{0} ; \omega_{0},-\omega_{0}, \omega_{0}\right) \mid \mathbf{E}(\mathbf{r}, \mathrm{t}) \mathbf{E}^{*}(\mathbf{r}, \mathrm{t}) \mathbf{E}(\mathbf{r}, \mathrm{t}),
$$

where | indicates the normal tensor product for the mode fields. $\chi^{(3)}$ is a rank four tensor and all its components depends on the class of symmetry of the crystal. Let's notice that Eq. (3.18) does not have the sum-frequency generation term because we have assumed that this process is not phasematched. Here, we have also assumed that the optical wave in the medium has a frequency equal to that of the incident wave. As silicon belongs to the crystallographic point group $\mathrm{m3m}$. Therefore the electronic nonlinear response $\chi^{(3)}$ has 21 nonzero elements, of which only 4 are independent, namely $\chi_{x x y y}^{(3)}, \chi_{x y x y}^{(3)}, \chi_{x y y x}^{(3)}$ and $\chi_{x x x x}^{(3)}$. Their intrinsic permutation symmetry allows us to simplify considerably, and we obtain [2] [11] 


$$
\chi_{\mu \alpha \beta \gamma}^{(3)}=\chi_{x x x x}^{(3)}\left[\frac{\rho}{3}\left(\delta_{\mu \alpha} \delta_{\beta \gamma}+\delta_{\mu \beta} \delta_{\alpha \gamma}+\delta_{\mu \gamma} \delta_{\alpha \beta}\right)+(1-\rho) \delta_{\mu \alpha \beta \gamma}\right],
$$

where $\rho \equiv 3 \chi_{x x y y}^{(3)} / \chi_{x x x x}^{(3)}$ represents the nonlinearity anisotropy at the degenerate frequency $\omega_{0}$ and the complex parameter $\chi_{x x x x}^{(3)}$ is related to the Kerr coefficient $n_{2}$ and the TPA coefficient $\beta_{T}$ as [5]

$$
\frac{\omega_{0}}{c} n_{2}\left(\omega_{0}\right)+\frac{i}{2} \beta_{T}\left(\omega_{0}\right)=\frac{3 \omega_{0}}{4 \varepsilon_{0} c^{2} n_{0}^{2}\left(\omega_{0}\right)} \chi_{x x x x}^{(3)}
$$

where $n_{0}$ is the linear refractive index of silicon at the frequency $\omega_{0}$. Henceforth, the Greek subscripts are used for denoting the Cartesian components of the vectors.

Equation (3.19) is written in a coordinate system, whose axes do not generally coincide with the crystallographic axes of the waveguide. Therefore to obtain the nonlinear response in our coordinate system we need to apply a suitable rotation of the basis to transform the tensor from the crystallographic coordinates into the coordinates of the waveguide. The general transformation for the nonlinear response tensor is given by [12]

$$
\chi_{\mu \alpha \beta \gamma}^{(3)}=\sum_{k \mathrm{lmn}} \mathrm{M}_{k \mu \mu} \mathrm{M}_{\mathrm{l} \alpha} \mathrm{M}_{\mathrm{m} \beta} \mathrm{M}_{\mathrm{m \gamma}} \chi_{\mathrm{klmn}}^{(3)},
$$

where $M$ is the transformation matrix. Now, if we notice that the only term involving $\delta_{\mu \alpha \beta \gamma}$ is rotation variant, then Eq. (3.19) in the rotated coordinate system becomes

$$
\chi_{\mu \alpha \beta \gamma}^{(3)}=\chi_{x x x x}^{(3)}\left[\frac{\rho}{3}\left(\delta_{\mu \alpha} \delta_{\beta \gamma}+\delta_{\mu \beta} \delta_{\alpha \gamma}+\delta_{\mu \gamma} \delta_{\alpha \beta}\right)+(1-\rho) \sum_{r=1}^{3} M_{r \mu} M_{r \alpha} M_{r \beta} M_{r y}\right] .
$$


SOI waveguides fabricated along the [110] crystallographic direction on the (100) wafer surface have been used in a lot of experiments in nonlinear optics [12], where the rotation angle $45^{\circ}$ and the transformation matrix is of the form

$$
M=\left(\begin{array}{ccc}
1 / \sqrt{2} & -1 / \sqrt{2} & 0 \\
1 / \sqrt{2} & 1 / \sqrt{2} & 0 \\
0 & 0 & 1
\end{array}\right)
$$

In the time domain, the form of the electric field in Eq. (3.8) has a carrier wave of the form $\exp \left[i\left(\beta z-\omega_{0} t\right)\right]$ with frequency $\omega_{0}$, and associated propagation constant $\beta$, i.e.,

$$
\mathbf{E}(\mathbf{r}, \mathrm{t}) \approx \sum_{\mathrm{m}=1}^{\mathrm{M}} a_{\mathrm{m}}(\mathrm{z}, \mathrm{t}) \frac{\mathbf{e}_{\mathrm{m}}(\mathrm{x}, \mathrm{y})}{\sqrt{\mathrm{N}_{\mathrm{m}}}} \mathrm{e}^{\mathrm{i}\left(\beta z-\omega_{0} \mathrm{t}\right)},
$$

where the modal amplitude $a_{\mathrm{m}}$ is related to the optical power. Therefore by substituting Eq. (3.24) into Eq. (3.18) and (3.16) and using them in Eq. (3.14), we obtain the following equation for the modal amplitudes:

$$
\begin{aligned}
\frac{\partial a_{\mathrm{k}}}{\partial \mathrm{z}}= & {\left[\sum_{\mathrm{n}=1}^{\infty} \mathrm{i}^{\mathrm{n}+1} \frac{\beta_{\mathrm{kn}}}{\mathrm{n} !} \frac{\partial^{\mathrm{n}}}{\partial \mathrm{t}^{\mathrm{n}}}\right] a_{\mathrm{k}}+\mathrm{i} \sum_{\mathrm{m}=1}^{\mathrm{M}} \kappa_{\mathrm{km}} a_{\mathrm{m}}+\mathrm{i} \delta_{\mathrm{km}} a_{\mathrm{k}} } \\
& +\mathrm{i} \sum_{\mathrm{h}} \frac{\omega_{0} \mathrm{n}_{0} \varepsilon_{0}}{2 \sqrt{N_{\mathrm{k}} N_{\mathrm{h}}}} a_{\mathrm{h}} \iint\left[\Delta \mathrm{n}^{\mathrm{f}}+\left(\mathrm{i} / 2 \mathrm{k}_{0}\right) \Delta \alpha^{\mathrm{f}}\right]\left(\mathbf{e}_{\mathrm{k}}^{*} \cdot \mathbf{e}_{\mathrm{h}}\right) \mathrm{dxdy} \\
& +\mathrm{i} \sum_{\mathrm{hnl}} \frac{3 \omega_{0} \varepsilon_{0}}{16 \sqrt{N_{\mathrm{k}} \mathrm{N}_{\mathrm{h}} \mathrm{N}_{\mathrm{n}} \mathrm{N}_{l}}} a_{\mathrm{h}} a_{\mathrm{n}}^{*} a_{1} \sum_{\mu \alpha \beta \gamma} \iint \chi_{\mu \alpha \beta \gamma}^{(3)} \mathrm{e}_{\mu, \mathrm{k}}^{*} \mathrm{e}_{\alpha, \mathrm{h}} \mathrm{e}_{\beta, \mathrm{n}}^{*} \mathrm{e}_{\gamma, \mathrm{l}} \mathrm{dxdy} .
\end{aligned}
$$

where the indices $k, h, n$ and I take values 1 to $M$ corresponding to the $M$ modes or nanowires of the array. This is the most general relation that govern the behavior of the modal amplitudes of the nanowires in the coupled system. Rewritten Eq. (3.25) in a compact form we obtain 


$$
\begin{aligned}
& \frac{\partial a_{\mathrm{k}}}{\partial \mathrm{z}}=\left[\sum_{\mathrm{n}=1}^{\infty} \mathrm{i}^{\mathrm{n}+1} \frac{\beta_{\mathrm{kn}}}{\mathrm{n} !} \frac{\partial^{\mathrm{n}}}{\partial \mathrm{t}^{\mathrm{n}}}\right] a_{\mathrm{k}}+\mathrm{i} \sum_{\mathrm{m}=1}^{\mathrm{M}} \kappa_{\mathrm{km}} a_{\mathrm{m}}+\mathrm{i} \delta_{\mathrm{km}} a_{\mathrm{k}}+\mathrm{i} \sum_{\mathrm{h}} \Lambda_{\mathrm{h}}^{(\mathrm{k})} \mathrm{N}_{\mathrm{h}}^{(\mathrm{k})} a_{\mathrm{h}} \\
&+\mathrm{i} \sum_{\mathrm{h}} \Omega_{\mathrm{h}}^{(\mathrm{k})} \mathrm{N}_{\mathrm{h}}^{(\mathrm{k})} a_{\mathrm{h}}+\mathrm{i} \sum_{\mathrm{hnl}} \gamma_{\mathrm{hnl}}^{(\mathrm{k})} a_{\mathrm{h}} a_{\mathrm{n}}^{*} a_{1}
\end{aligned}
$$

where we can identify the nonlinear parameter $\gamma_{h n l}^{(k)}, \Lambda_{h}^{(k)}$ and $\Omega_{h}^{(k)}$ given by

$$
\begin{gathered}
\gamma_{\mathrm{hnl}}^{(\mathrm{k})}=\frac{\mathrm{n}_{0}^{2} \mathrm{k}_{0} \mathrm{n}_{2}}{\overline{\mathrm{n}}^{2} \overline{\mathrm{A}}_{\text {eff }}} \Gamma_{\mathrm{hnl}}^{(\mathrm{k})} \eta_{\mathrm{hnl}}^{(\mathrm{k})}(1+\mathrm{ir}), \\
\Lambda_{\mathrm{h}}^{(\mathrm{k})}=\frac{\mathrm{n}_{0} \mathrm{k}_{0} \sigma_{\mathrm{n}}}{\overline{\mathrm{n}}^{\prime}} \Gamma_{\mathrm{h}}^{(\mathrm{k})}, \\
\Omega_{\mathrm{h}}^{(\mathrm{k})}=\mathrm{i} \frac{1}{2} \frac{\mathrm{n}_{0} \sigma_{a}}{\overline{\mathrm{n}}^{\prime}} \Gamma_{\mathrm{h}}^{(\mathrm{k})} .
\end{gathered}
$$

The additional parameters are given by

$$
\begin{aligned}
& \eta_{\mathrm{hnl}}^{(\mathrm{k})}=\frac{\sum_{\mu \alpha \beta \gamma} \iint \chi_{\mu \alpha \beta \gamma}^{(3)} \mathrm{e}_{\mu, \mathrm{k}}^{*} \mathrm{e}_{\alpha, \mathrm{h}} \mathrm{e}_{\beta, \mathrm{n}}^{*} \mathrm{e}_{\gamma, \mathrm{l}} \mathrm{dxdy}}{\chi_{x x \times x}^{(3)}\left[\prod_{v=k, h, h, l} \iint\left(\left|\mathbf{e}_{\mathrm{v}}\right|^{2}\right)^{2} \mathrm{dxdy}\right]^{1 / 4}} \\
& \bar{A}_{\text {eff }}=\left(\prod_{v=k, h, n, l} A_{\text {eff }}^{(v)}\right)^{1 / 4} \\
& \Gamma_{\mathrm{hnl}}^{(\mathrm{k})}=\frac{\left[\prod_{v=k, h, n, l}\left(\iint\left|\mathbf{e}_{v}\right|^{2} \mathrm{dxdy}\right)^{2}\right]^{1 / 4}}{\left(\prod_{v=k, n, n, l} \iint\left[\left|\mathbf{e}_{\mathrm{Tv}}\right|^{2}-\mathrm{i}\left(\beta^{\mathrm{v}}\right)^{-1}\left(\mathbf{e}_{\mathrm{Tv}} \cdot \nabla_{\mathrm{T}} \mathrm{e}_{\mathrm{zv}}^{*}\right)\right] \mathrm{dxdy}\right)^{1 / 2}} \\
& \overline{\mathrm{n}}^{2}=\left(\prod_{v=k, h, n, l} \overline{\mathrm{n}}_{\mathrm{v}}\right)^{1 / 2}
\end{aligned}
$$




$$
\begin{gathered}
\mathrm{N}_{\mathrm{h}}^{(\mathrm{k})}=\frac{\iint \mathrm{N}\left(\mathbf{e}_{\mathrm{k}}^{*} \cdot \mathbf{e}_{\mathrm{h}}\right) \mathrm{dxdy}}{\left[\prod_{v=k, \mathrm{~h}}\left(\iint\left|\mathbf{e}_{v}\right|^{2} \mathrm{~d} x \mathrm{~d} d \mathrm{y}\right)^{2}\right]^{1 / 4}} \\
\overline{\mathrm{n}}^{\prime}=\left(\prod_{v=k, \mathrm{~h}} \overline{\mathrm{n}}_{\mathrm{v}}\right)^{1 / 2}
\end{gathered}
$$

where $\bar{n}_{k}=\beta_{k} / k_{0}$ represents the mode index and the nonlinear effective mode area $A_{\text {eff }}$ of the mode $k$ is defined as [6]

$$
A_{\text {eff }}^{(k)}=\frac{\left(\iint\left|\mathbf{e}_{k}\right|^{2} d x d y\right)^{2}}{\iint\left(\left|\mathbf{e}_{k}\right|^{2}\right)^{2} d x d y} .
$$

Note that $A_{\text {eff }}$ depends on the waveguide parameters such as the cross section of the core. Because $A_{\text {eff }}$ is quite small for silicon nanowires, exist an enhancement of the nonlinear effects in them. For the fundamental modes on silicon nanowires, the effective mode area $A_{\text {eff }}$ has a value which is similar, but not identical, to the cross-sectional area of the nanowire because the intensity is not zero totally outside the waveguide. Note that Eq. (3.30) represents the average of the density of free-carriers over the effective mode area. If we assume that free-carriers are generated exclusively by TPA, and that all carrier recombination processes can be described by a single time constant, then the average value of the free-carrier density of free electrons and holes, $\mathrm{N}_{h}^{(k)}$, is governed by the following equation of the form [2]

$$
\frac{\mathrm{dN}_{\mathrm{h}}^{(\mathrm{k})}}{\mathrm{dt}}=\mathrm{G}-\frac{\mathrm{N}_{\mathrm{h}}^{(\mathrm{k})}}{\tau_{0}}
$$


where $\tau_{0}$ is the free-carrier lifetime and $G$ is the generation rate. The above equation shows the rate of electron-hole pair generation and recombination inside the waveguide. The evaluation of the previous parameters requires the use of the modal distribution $\mathbf{e}$ for the fundamental mode of each nanowire.

If the single-mode nanowires of the array have the same nonlinear constants and thicknesses, the Kerr effect in all the nanowires will be the same when the same optical power is launched into any waveguide. Furthermore, we will assume that are coupled by superposition only with the closest adjacent waveguides and any others coupling can be neglected. The constant coupling coefficient between the $k$ and $m$ waveguides is given by $\kappa_{\mathrm{km}}$. Consequently, if we use identical nanowires, the coupling coefficients between the $m$ waveguide and any photonic wire will be equal, i.e. $\kappa_{\mathrm{km}}=\kappa_{\mathrm{mk}}$ $[5,9]$. In this particular case, $m$ will be able to take the values $k-1$ and $k+1$. Because of the superposition interaction, there will be a contribution to the kth mode from all the superposition coupling that will contribute to the loss and the phase of the kth mode. Such contribution is expressed as a self coupling in our equations.

$$
\begin{aligned}
& \frac{\partial a_{\mathrm{k}}}{\partial \mathrm{z}}+\frac{\alpha_{\mathrm{k}}}{2} a_{\mathrm{k}}=\left[\sum_{\mathrm{n}=1}^{\infty} \mathrm{i}^{\mathrm{n}+1} \frac{\beta_{\mathrm{kn}}}{\mathrm{n} !} \frac{\partial^{\mathrm{n}}}{\partial \mathrm{t}^{\mathrm{n}}}\right] a_{\mathrm{k}}+\mathrm{i} \sum_{\mathrm{m}=\mathrm{k}-1}^{k+1} \kappa_{\mathrm{km}} a_{\mathrm{m}} \\
&+\mathrm{i}[\left.\mathrm{Q}_{1}\left|a_{\mathrm{k}}\right|^{2}+2 \mathrm{Q}_{2} \sum_{\mathrm{w}=\mathrm{k}-1, \mathrm{k}+1} \sum_{\mathrm{v}=\mathrm{k}-1, \mathrm{k}+1} a_{\mathrm{w}} a_{\mathrm{v}}^{*}\right] a_{\mathrm{k}} \\
&+\mathrm{i}\left[\mathrm{Q}_{4} \sum_{\mathrm{v}=\mathrm{k}-1, \mathrm{k}+1} \sum_{\mathrm{w}=\mathrm{k}-1, \mathrm{k}+1} \sum_{\mathrm{v}=\mathrm{k}-1, \mathrm{k}+1} a_{\mathrm{u}} a_{\mathrm{w}} a_{\mathrm{v}}^{*}+2 \mathrm{Q}_{3} \sum_{\mathrm{v}=\mathrm{k}-1, \mathrm{k}+1} a_{\mathrm{v}}\left|a_{\mathrm{k}}\right|^{2}\right] \\
&+\mathrm{i}\left[\mathrm{Q}_{5} \sum_{\mathrm{w}=\mathrm{k}-1, \mathrm{k}+1} \sum_{\mathrm{v}=\mathrm{k}-1, \mathrm{k}+1} a_{\mathrm{w}} a_{\mathrm{v}} a_{\mathrm{k}}^{*}+\mathrm{Q}_{6} \sum_{\mathrm{v}=\mathrm{k}-1, \mathrm{k}+1} a_{\mathrm{k}}^{2} a_{\mathrm{v}}^{*}\right] \\
&+\mathrm{i} \Lambda_{\mathrm{k}}^{(\mathrm{k})} \mathrm{N}_{\mathrm{k}}^{(\mathrm{k})} a_{\mathrm{k}}+\mathrm{i} \Omega_{\mathrm{k}}^{(\mathrm{k})} \mathrm{N}_{\mathrm{k}}^{(\mathrm{k})} a_{\mathrm{k}}
\end{aligned}
$$


We should stress here that $\mathrm{u}, \mathrm{w}, \mathrm{v}=\mathrm{k}-1$ or $\mathrm{k}+1$, the $\alpha_{\mathrm{k}}$ term have been added heuristically to account for internal waveguides linear attenuations and the self coupling has been already explained from the near neighbor superposition. The terms due to the free-carriers of the adjacent nanowires are neglected. The nonlinear coefficients $Q_{1}$ to $Q_{6}$ are given by:

- $\mathrm{Q}_{1}=\gamma_{\mathrm{kkk}}^{(\mathrm{k})}$,

- $\mathrm{Q}_{2}=\gamma_{\mathrm{k}-1, \mathrm{k}-1, \mathrm{k}}^{(\mathrm{k})}=\gamma_{\mathrm{k}, \mathrm{k}-1, \mathrm{k}-1}^{(\mathrm{k})}=\gamma_{\mathrm{k}+1, \mathrm{k}+1, \mathrm{k}}^{(\mathrm{k})}=\gamma_{\mathrm{k}, \mathrm{k}+1, \mathrm{k}+1}^{(\mathrm{k})}=\gamma_{\mathrm{k}-1, \mathrm{k}+1, \mathrm{k}}^{(\mathrm{k})}=\gamma_{\mathrm{k}, \mathrm{k}+1, \mathrm{k}-1}^{(\mathrm{k})}$ $=\gamma_{k+1, k-1, k}^{(k)}=\gamma_{k, k-1, k+1}^{(k)}$

- $\mathrm{Q}_{3}=\gamma_{\mathrm{k}-1, \mathrm{k}, \mathrm{k}}^{(\mathrm{k})}=\gamma_{\mathrm{k}, \mathrm{k}, \mathrm{k}-1}^{(\mathrm{k})}=\gamma_{\mathrm{k}+1, \mathrm{k}, \mathrm{k}}^{(\mathrm{k})}=\gamma_{\mathrm{k}, \mathrm{k}, \mathrm{k}+1}^{(\mathrm{k})}$,

- $\mathrm{Q}_{4}=\gamma_{\mathrm{k}-1, \mathrm{k}-1, \mathrm{k}-1}^{(\mathrm{k})}=\gamma_{\mathrm{k}+1, \mathrm{k}+1, \mathrm{k}+1}^{(\mathrm{k})}=\gamma_{\mathrm{k}+1, \mathrm{k}-1, \mathrm{k}-1}^{(\mathrm{k})}=\gamma_{\mathrm{k}-1, \mathrm{k}+1, \mathrm{k}+1}^{(\mathrm{k})} \gamma_{\mathrm{k}-1, \mathrm{k}-1, \mathrm{k}+1}^{(\mathrm{k})}=\gamma_{\mathrm{k}+1, \mathrm{k}+1, \mathrm{k}-1}^{(\mathrm{k})}$ $=\gamma_{\mathrm{k}+1, \mathrm{k}-1, \mathrm{k}+1}^{(\mathrm{k})}=\gamma_{\mathrm{k}-1, \mathrm{k}+1, \mathrm{k}-1,}^{(\mathrm{k})}$

- $\mathrm{Q}_{5}=\gamma_{\mathrm{k}-1, k, k-1}^{(\mathrm{k})}=\gamma_{\mathrm{k}+1, k, k+1}^{(\mathrm{k})}=\gamma_{\mathrm{k}-1, k, k+1}^{(\mathrm{k})}=\gamma_{\mathrm{k}+1, k, k-1}^{(\mathrm{k})}$,

- $\mathrm{Q}_{6}=\gamma_{\mathrm{k}, \mathrm{k}+1, \mathrm{k}}^{(\mathrm{k})}=\gamma_{\mathrm{k}, \mathrm{k}-1, \mathrm{k}}^{(\mathrm{k})}$.

In general, we can notice that the linear contributions to the coupling in Eq. (3.33) arise from the overlap of the mode field with an adjacent waveguide and from the presence of any mode of the adjacent guide. Nonlinear contributions arise due to the mode interaction with the material by itself or in conjunction with a mode from the adjacent waveguide.

In particular, we are interested in an array that consists of two identical silicon photonic wires in close proximity; the coupled-mode equations obtained for this case are given by 


$$
\begin{aligned}
& \frac{\partial a_{1}}{\partial \mathrm{z}}+\frac{\alpha_{1}}{2} a_{1}=\left[\sum_{\mathrm{n}=1}^{\infty} \mathrm{i}^{\mathrm{n}+1} \frac{\beta_{\mathrm{n}}}{\mathrm{n} !} \frac{\partial^{\mathrm{n}}}{\partial \mathrm{t}^{\mathrm{n}}}\right] a_{1}+\mathrm{i} \kappa_{11} a_{1}+\mathrm{i}_{\kappa_{12}} a_{2}+\mathrm{iQ}_{1}\left(\left|a_{1}\right|^{2}+2\left|a_{2}\right|^{2}\right) a_{1} \\
& +\mathrm{iQ}_{3}\left(\left|a_{2}\right|^{2}+2\left|a_{1}\right|^{2}\right) a_{2}+\mathrm{iQ} a_{2} a_{1}^{*}+\mathrm{iQ}_{3} a_{1}^{2} a_{2}^{*}+\mathrm{i} \Lambda \bar{N} a_{1}+\mathrm{i} \Omega \bar{N} a_{1} \\
& \frac{\partial a_{2}}{\partial \mathrm{z}}+\frac{\alpha_{2}}{2} a_{2}=\left[\sum_{\mathrm{n}=1}^{\infty} \mathrm{i}^{\mathrm{n}+1} \frac{\beta_{\mathrm{n}}}{\mathrm{n} !} \frac{\partial^{\mathrm{n}}}{\partial \mathrm{t}^{\mathrm{n}}}\right] a_{2}+\mathrm{i} \kappa_{22} a_{2}+\mathrm{i} \kappa_{12} a_{1}+\mathrm{iQ}_{1}\left(\left|a_{2}\right|^{2}+2\left|a_{1}\right|^{2}\right) a_{2} \\
& +\mathrm{iQ}_{3}\left(\left|a_{1}\right|^{2}+2\left|a_{2}\right|^{2}\right) a_{1}+\mathrm{iQ} a_{1}^{2} a_{2}^{*}+\mathrm{iQ}_{3} a_{2}^{2} a_{1}^{*}+\mathrm{i} \Lambda \overline{\mathrm{N}} a_{2}+\mathrm{i} \Omega \bar{N} a_{2}
\end{aligned}
$$

where $\quad \mathrm{Q}_{3}=\mathrm{Q}_{4}=\mathrm{Q}_{6}, \quad \mathrm{Q}_{1}=\mathrm{Q}_{2}=\mathrm{Q}_{5}, \quad \mathrm{~N}_{1}^{(1)}=\mathrm{N}_{2}^{(2)}=\overline{\mathrm{N}}, \quad \Lambda_{1}^{(1)}=\Lambda_{2}^{(2)}=\Lambda \quad$ and $\Omega_{1}^{(1)}=\Omega_{2}^{(2)}=\Omega$ for the symmetric form. We have used the symmetry in the coupling coefficients $\kappa_{12}=\kappa_{21}$ and have assumed that the self coupling $\kappa_{11}=\kappa_{22}$ is identical as well. These equations are an extended version of the Nonlinear Schrodinger equation used for single photonic wires [5]. The previous equations are the most general form of the coupled-mode equations for two coupled silicon nanowires. Let's identify each term of Eq. (3.34), for example, we can notice that

- $\kappa_{\mathrm{ii}}$ modify the propagation constant of the mode $\mathrm{i}$, the

- $\kappa_{\mathrm{ij}}$ term led to a linear coupling between the modes of the waveguides $\mathrm{i}$ and $\mathrm{j}$.

The first term in the first parenthesis involving

- $\mathrm{Q}_{1}$ is the strongest nonlinear term and arise from the nonlinear interaction of a mode with itself.

The remaining parameters describe the self-phase modulation and the twophoton absorption effect. 
- The second term in the same parenthesis involving $\mathrm{Q}_{1}$ arises from the nonlinear interaction of one mode with the mode in the adjacent waveguide. This term implicate the cross-phase modulation (XPM).

- The terms in the second parenthesis involving $\mathrm{Q}_{3}$ arises from the power-dependent linear coupling coefficients and

- The last two terms, with $\Lambda$ and $\Omega$, arise from the nonlinear coupling. The same analysis can be done for Eq. (3.35).

In the next section, we are going to study some cases for the propagation of light through the array which help us to understand the nonlinear phenomena in the coupled structures and its applications to the optical processing.

\subsection{Summary}

In this chapter a theoretical model is developed for describing the evolution of an optical signal through $\mathrm{N}$ coupled silicon waveguides. $\mathrm{A}$ generalized theoretical analysis of the nonlinear coupled system was given where we have considered the vector nature of optical modes and the effects of TPA on various nonlinear processes. The general theoretical model includes not only the instantaneous nonlinear processes and free-carrier effects but also linear and nonlinear losses, and it extends previous vector nonlinear models to the case where coupling of supermodes of a waveguide array occurs in silicon waveguides. The material presented here was deduced for the first time, although it is based in some investigations already done in the literature $[1,2]$. 


\section{References}

[1] B. A. Daniel and G. P. Agrawal, "Vectorial nonlinear pulse propagation in silicon nanowire waveguides: polarization effects," J. Opt. Soc. Am. B 27, 956-965 (2010).

[2] Q. Lin, O. J. Painter, and G. P. Agrawal, "Nonlinear optical phenomena in silicon waveguides: modeling and applications," Opt. Express 15, 1660416644 (2007).

[3] L. Pavesi and D. J. Lockwood, Eds., Silicon Photonics (Springer, New York, 2004).

[4] R. M, Osgood, Jr., N C. Panoiu, J. I. Dadap, X. Liu, X. Chen, I. W. Hsieh, E. Dulkeith, W. M. Green, and Y. A. Vlasov, "Engineering nonlinearities in nanoscale optical systems: physiscs and applications in dispersionengineered silicon nanophotonic wires," Adv. Opt. Photonics. 1, 162-235 (2009).

[5] G. P. Agrawal, Nonlinear Fiber Optics (Academic, 2007).

[6] S. Afshar V. and T. M. Monro, "A full vectorial model for pulse propagation in emerging waveguides with subwavelength structures part 1: Kerr nonlinearity," Opt. Express 17, 2298-2318 (2009).

[7] A. W. Snyder and J. D. Love, Optical Waveguide Theory, (Kluwer Academic, 2000).

[8] R. W. Boyd, Nonlinear Optics, $2^{\text {nd }}$ ed. (Academic Press, Boston, 2003).

[9] A. Hardy and W. Streifer, J. Lightwave Technol., vol. LT-3, pp. 11351146, 1985.

[10] G. P. Agrawal, Applications of Nonlinear Fiber Optics, $2^{\text {nd }}$ ed. (Academic, 2008).

[11] P. N. Butcher and D. Cotter, The Elements of Nonlinear Optics, (Cambridge University Press, 1990). 
[12] S. M. Jensen, IEEE J. Quantum Electron., vol. QE-18, pp. 1580-1583, 1982

[13] S. M. Jensen, IEEE J. Quantum Electron., vol. QE-18, pp. 1580-1583, 1982 


\section{Chapter 4}

\section{Nonlinear Propagation in Coupled}

\section{Silicon Nanowires}

Nonlinear propagation of optical pulses inside coupled silicon nanowires is theoretically investigated. Output powers relations are derived for some cases in which propagation of picoseconds pulses is considered concerning the initial switching condition. Input-output power relations account for linear losses, the Kerr nonlinearity, TPA, FCA and FCD, linear coupling, and the power-dependent coupling effect. This work can be considered as a generalization of some investigations already done in literature (see for example Amarendra [1]). In particular, nonlinear processes, TPA, FCA, FCD and power-dependent coupling effects on the formation and propagation of optical pulses inside coupled silicon nanowires are investigated. Based on our study, we generalize the standard definition of coupling length for our nonlinear directional coupler according to the catch-all phase difference. As a consequence of the previous theoretical work (see chapter 3), in section 4.1 we reintroduce the general coupled system of differential nonlinear equations that govern the picosecond pulse evolution inside two coupled silicon nanowires. Before addressing the most general problem, which is planned for the future, in this work we consider some practically important cases in which the dispersion on the one hand (Section 4.1 .1 and 4.1.2) and free-carriers on 
the other hand (Section 4.1.1 and 4.1.3) are neglected. Soliton formation and propagation are presented and discussed in all cases. It can be easily shown that this scenario is achieved by a new complex amplitude reformulation.

\subsection{Coupled Nonlinear Equations}

In the previous chapter, we have obtained a set of general equations that provide an understanding and theoretical description of the nonlinear response of coupled silicon waveguides. In this section, we introduce an interesting new form of the coupled evolution equations that govern the pulse dynamics in such a coupled silicon nanowires. Raman scattering is excluded in all the work because we consider the pulse bandwidth shorter than the Raman shift (15.1 THz for silicon [2]). Therefore, this investigation lies under the picosecond regime and we neglect the higher order nonlinear effects induced by the delayed Raman response. Since in a single-mode silicon waveguide two propagating modes are typically confined, one quasiTE and one quasi-TM, this work is only referred to one of them as polarized input signals.

Consider a pulse signal at a carrier frequency $\omega_{0}$ propagating through the coupled system, as shown in Fig. 4.1 (a). By using the theoretical model given by Eqs. (3.32), (3.34) and (3.35), the longitudinal evolution of a electric field $\mathbf{E}$ associated with this optical pulse signal is described by following coupled nonlinear equations:

$$
\begin{aligned}
\frac{\partial a_{1}}{\partial \mathrm{z}}+ & \beta_{1} \frac{\partial a_{1}}{\partial \mathrm{t}}+\frac{\mathrm{i} \beta_{2}}{2} \frac{\partial^{2} a_{1}}{\partial \mathrm{t}^{2}}+\frac{\alpha}{2} a_{1}=\mathrm{i} \kappa_{1} a_{1}+\mathrm{i}\left[\kappa_{2}+\mathrm{Q}_{3}\left(\left|a_{2}\right|^{2}+2\left|a_{1}\right|^{2}\right)\right] a_{2} \\
& +\mathrm{i} \mathrm{Q}_{1}\left(\left|a_{1}\right|^{2}+2\left|a_{2}\right|^{2}\right) a_{1}+\mathrm{i} \mathrm{Q}_{1} a_{2}^{2} a_{1}^{*}+\mathrm{i} \mathrm{Q}_{3} a_{1}^{2} a_{2}^{*}-\frac{\sigma}{2}(1+\mathrm{i} \mu) \overline{\mathrm{N}} a_{1}
\end{aligned}
$$




$$
\begin{gathered}
\frac{\partial a_{2}}{\partial \mathrm{z}}+\beta_{1} \frac{\partial a_{2}}{\partial \mathrm{t}}+\frac{\mathrm{i} \beta_{2}}{2} \frac{\partial^{2} a_{2}}{\partial \mathrm{t}^{2}}+\frac{\alpha}{2} a_{2}=\mathrm{i} \kappa_{1} a_{2}+\mathrm{i}\left[\kappa_{2}+\mathrm{Q}_{3}\left(\left|a_{1}\right|^{2}+2\left|a_{2}\right|^{2}\right)\right] a_{1} \\
+\mathrm{iQ}_{1}\left(\left|a_{2}\right|^{2}+2\left|a_{1}\right|^{2}\right) a_{2}+\mathrm{iQ} a_{1}^{2} a_{2}^{*}+\mathrm{iQ} a_{2}^{2} a_{1}^{*}-\frac{\sigma}{2}(1+\mathrm{i} \mu) \overline{\mathrm{N}} a_{2} \\
\frac{\partial \overline{\mathrm{N}}}{\partial \mathrm{t}}=\frac{\beta_{\mathrm{T}}}{2 \mathrm{~h} \omega_{0} \mathrm{~A}_{\text {eff }}^{2}}\left(\left|a_{1}\right|^{2}+\left|a_{2}\right|^{2}\right)^{2}-\frac{\overline{\mathrm{N}}}{\tau_{0}},
\end{gathered}
$$

that describe the coupled modes and the free-carrier density of free electrons and holes $\bar{N}$. The parameters $\beta_{1}$ and $\beta_{2}$ have their origin in the frequency dependence of the propagation constant, and we have assumed symmetry in the coupling $\kappa_{21}=\kappa_{12}=\kappa_{2}$ and the same self-modulation $\kappa_{11}=\kappa_{22}=\kappa_{1}$. Here, the parameters $\sigma$ and $\mu$ are given by

$$
\sigma=\sigma_{\mathrm{n}}\left(\frac{\mathrm{n}_{0}}{\overline{\bar{n}^{\prime}}} \Gamma\right)\left(\frac{\lambda_{0}}{\lambda_{\mathrm{r}}}\right) \quad \mu=2 \mathrm{k}_{0} \frac{\sigma_{n}}{\sigma_{a}}
$$

where $\sigma_{a}=1.45 \times 10^{-21} \mathrm{~m}^{2}$ is the FCA coefficient at the reference wavelength $\lambda_{\mathrm{r}}=1550 \mathrm{~nm}$, and the changes in the refractive index by free-carriers are given by $\mu$, where $\sigma_{\mathrm{n}}=5.3 \times 10^{-27} \mathrm{~m}^{3}$ [9].

Lets notice that Eqs. (4.1)-(4.3) describe the time-space evolution of both the quasi-TE or the quasi-TM modes, also that the simultaneous solution of these three coupled nonlinear partial differential equations will determine $\overline{\mathrm{N}}(\mathrm{z}, \mathrm{t}), a_{1}(\mathrm{z}, \mathrm{t})$ and $a_{2}(\mathrm{z}, \mathrm{t})$. We have assumed that the complex pulse amplitudes $a_{1}$ and $a_{2}$, while deriving Eqs. (4.1), (4.2) and (4.3), are normalized such that $\left|a_{1}\right|^{2}$ and $\left|a_{2}\right|^{2}$ represent optical powers. These equations have a complex mathematical structure, however, they can be readily solved by using the even and odd eigenmodes of the nonlinear 
directional coupler (also called supermodes). For this purpose, we introduce two new complex variables

$$
\begin{aligned}
& \mathrm{b}_{1}=\left(\frac{a_{1}+a_{2}}{2}\right) \mathrm{e}^{-\mathrm{i} \mathrm{K}_{1} \mathrm{z}} \\
& \mathrm{b}_{2}=\left(\frac{a_{1}-a_{2}}{2}\right) \mathrm{e}^{-\mathrm{i} \mathrm{K}_{1} \mathrm{z}} .
\end{aligned}
$$

These two specific linear combinations of $a_{1}$ and $a_{2}$ constitute the amplitudes associated with the even and odd supermodes of the nonlinear directional coupler. Before strategy makes the equations analytically tractable. In terms of the new complex amplitudes, $b_{1}$ and $b_{2}$, Eqs. (4.1), (4.2) and (4.3) can be rewritten as:

$$
\begin{gathered}
\frac{\partial \mathrm{b}_{1}}{\partial \mathrm{z}}+\beta_{1} \frac{\partial \mathrm{b}_{1}}{\partial \mathrm{t}}+\frac{\mathrm{i} \beta_{2}}{2} \frac{\partial^{2} \mathrm{~b}_{1}}{\partial \mathrm{t}^{2}}+\frac{\alpha}{2} \mathrm{~b}_{1}=\mathrm{i}_{2} \mathrm{~b}_{1}+i \mathrm{C}_{1} \mathrm{~b}_{1}\left|\mathrm{~b}_{1}\right|^{2}-\frac{\sigma}{2}(1+\mathrm{i} \mu) \overline{\mathrm{N}} \mathrm{b}_{1} \\
\frac{\partial \mathrm{b}_{2}}{\partial \mathrm{z}}+\beta_{1} \frac{\partial \mathrm{b}_{2}}{\partial \mathrm{t}}+\frac{\mathrm{i} \beta_{2}}{2} \frac{\partial^{2} \mathrm{~b}_{2}}{\partial \mathrm{t}^{2}}+\frac{\alpha}{2} \mathrm{~b}_{2}=-\mathrm{i} \kappa_{2} \mathrm{~b}_{2}+i \mathrm{C}_{2} \mathrm{~b}_{2}\left|\mathrm{~b}_{2}\right|^{2}-\frac{\sigma}{2}(1+\mathrm{i} \mu) \overline{\mathrm{N}} \mathrm{b}_{2} \\
\frac{\partial \overline{\mathrm{N}}}{\partial \mathrm{t}}=\frac{2 \beta_{\mathrm{T}}}{\mathrm{h} \omega_{0} \mathrm{~A}_{\text {eff }}^{2}}\left(\left|\mathrm{~b}_{1}\right|^{2}+\left|\mathrm{b}_{2}\right|^{2}\right)^{2}-\frac{\overline{\mathrm{N}}}{\tau_{0}}
\end{gathered}
$$

where $C_{1}=4\left(Q_{1}+Q_{3}\right)$ and $C_{2}=4\left(Q_{1}-Q_{3}\right)$. On this reformulation, it can be easily shown that the even and odd supermodes are uncoupled linearly and coupled nonlinearly from the free-carrier terms. Phase velocities of the supermodes are not the same, as evident from different signs of the $\kappa_{2}$ term in the above equations. We may say that this set of supermodes coupled nonlinear equations, Eqs. (4.7), (4.8) and (4.9), have only one direct nonlinear term that corresponds to self-phase modulation (SPM) and some indirect nonlinear terms given by the free-carrier effect. 
Equations (4.7), (4.8) and (4.9) can be rewritten in terms of the soliton units such as $T_{0}$ is the width of the incident pulse and $P_{0}$ the pulse peak power. If we introduce dimensionless quantities such as $b_{1}=U_{1} \sqrt{P_{0}}$, $\mathrm{b}_{2}=\mathrm{U}_{2} \sqrt{\mathrm{P}_{0}}, \quad \mathrm{z}=\xi \mathrm{L}_{\mathrm{NL}}$ and $\tau=\mathrm{T} / \mathrm{T}_{0}$, where $\mathrm{L}_{\mathrm{NL}}=1 / \gamma_{0} \mathrm{P}_{0}$ is the nonlinear length, $\gamma_{0}=n_{2} \omega_{0} / c A_{\text {eff }}$ is the nonlinear parameter, $T=t-z \beta_{1}$ is the retarded time in the reference frame of the pulse, $\mathrm{T}_{0}$ is the width of the incident pulse and $P_{0}$ is the pulse peak power, Eqs. (4.7), (4.8) and (4.9) can be rewritten in the following dimensionless form:

$$
\begin{gathered}
\frac{\partial \mathrm{U}_{1}}{\partial \xi}+i \frac{\operatorname{sgn}\left(\beta_{2}\right)}{2 N^{2}} \frac{\partial^{2} U_{1}}{\partial \tau^{2}}+\left(\alpha_{L N}-i \kappa\right) U_{1}=i \frac{C_{1}}{\gamma_{0}} U_{1}\left|U_{1}\right|^{2}-\frac{\sigma}{2}(1+i \mu) L_{N L} \bar{N} U_{1} \\
\frac{\partial U_{2}}{\partial \xi}+i \frac{\operatorname{sgn}\left(\beta_{2}\right)}{2 N^{2}} \frac{\partial^{2} U_{2}}{\partial \tau^{2}}+\left(\alpha_{L N}+i \kappa\right) U_{2}=i \frac{C_{2}}{\gamma_{0}} U_{2}\left|U_{2}\right|^{2}-\frac{\sigma}{2}(1+i \mu) L_{N L} \bar{N} U_{2} \\
\frac{\partial \bar{N}}{\partial \tau}=\frac{2 P_{0}^{2} \beta_{T}}{h \omega_{0} A_{\text {eff }}^{2}}\left(\left|U_{1}\right|^{2}+\left|U_{2}\right|^{2}\right)^{2}-\frac{\bar{N}}{\tau_{0}}
\end{gathered}
$$

where $N^{2}=L_{D} / L_{N L}, \quad \kappa=\kappa_{2} L_{N L}, \quad \alpha_{L N}=\alpha L_{N L} / 2, L_{D}=T_{0}^{2} /\left|\beta_{2}\right|$ is the dispersion length and $\operatorname{sgn}\left(\beta_{2}\right)= \pm 1$ depending on the sign of the GVD parameter $\beta_{2}$. In fact, to analyze the switching process, we always consider as the initial conditions the pumping on only one core of the coupler by an external source of light, which is a special case of interest to optical data processing [3], such that $\mathrm{U}_{1}(0, \tau)=\mathrm{U}_{2}(0, \tau)$ and $\left|a_{1}(0, \tau)\right|^{2}=4 \mathrm{P}_{0}\left|\mathrm{U}_{1}(0, \tau)\right|^{2}$.

The proposed nonlinear directional coupler described here has two identical low-loss single-mode silicon nanowires placed close to each other throughout its length. The waveguides are close enough that the fundamental modes propagating in each nanowire overlap partially in the 
cladding region between the two waveguides. Such evanescent-wave coupling between the tails of two modes leads to the transfer of optical power from one waveguide to another under suitable conditions [4]. A scheme of the coupler cross-section is shown in Fig. 5 (b).

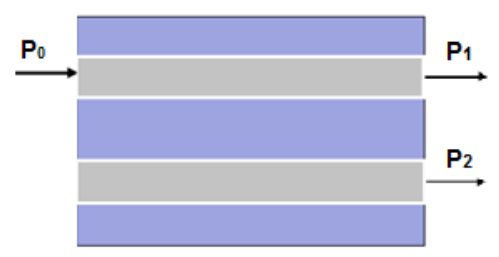

(a)

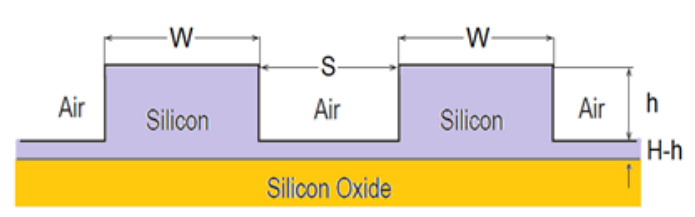

(b)

Figure 5. Coupled silicon nanowires design: (a) schematic illustration of the four-port device; (b) scheme of the cross-section.

The Design of silicon waveguide based nonlinear directional coupler is based on the waveguide characteristics, in particular the width $\mathrm{W}$, the height $\mathrm{H}$ and the etch depth $h$, and on the separation between the waveguides. The linear coupling coefficient $\kappa_{2}$ is completely determinate by this. In our schematic illustration the core-to-core distant is given by S. Ultimately, the separation between the waveguides is the utmost standard characteristic for the switching behavior. However, we will see later that the switching process is changed substantially by the TPA, the free-carriers-induced absorption and the nonlinear coupling coefficient given by $\mathrm{Q}_{3}$. The Power-dependent coupling terms, as small nonlinear terms, has been studied numerically for symmetric directional couplers [5], however, they do not provide an intuitive understanding for the switching process and nonlinear pulse propagation. This result provides a new definition of the coupling length for each case where we discuss, in detail, the soliton switching in the anomalous dispersion regime. 


\subsubsection{Nonlinear Effects}

Previous to the consideration of the general nonlinear pulse propagation in coupled silicon nanowires in picosecond regime, we will focus on a special case in which the dispersive and free-carrier terms can be neglected in Eqs. (4.10) and (4.11). This situation occurs when the coupler length is typically much shorter than the dispersion length $L_{D}$, the pulses repetition rate $R_{m}$ is low $\left(R_{m} \tau_{0} \square 1\right)$ and their peak intensity is not too high. In this case, the freecarrier density $\bar{N}$ is low enough that do not affect the pulse propagation and the input peak intensity $P_{0}$ satisfies the condition $P_{0} \square 3 h \omega_{0} A_{\text {eff }} /\left(\sigma T_{0}\right)[6]$. we arrive to the following set of coupled nonlinear partial differential equations, if we discard the dispersive and the free-carrier terms in the Eqs. (4.10) and (4.11),

$$
\begin{aligned}
& \frac{\partial \mathrm{u}_{1}}{\partial \xi}=\mathrm{i} \kappa \mathrm{u}_{1}+\mathrm{ie}^{-2 \alpha_{\llcorner\mathrm{s}}} \frac{\mathrm{C}_{1}}{\gamma_{0}} \mathrm{u}_{1}\left|\mathrm{u}_{1}\right|^{2} \\
& \frac{\partial u_{2}}{\partial \xi}=-i \kappa u_{2}+i e^{-2 \alpha_{L N} \xi} \frac{C_{2}}{\gamma_{0}} u_{2}\left|u_{2}\right|^{2} \text {, }
\end{aligned}
$$

where the new complex amplitudes are given by $U_{1}=u_{1} \exp \left(-\alpha_{L N} \xi\right)$, $\mathrm{U}_{2}=\mathrm{u}_{2} \exp \left(-\alpha_{\mathrm{LN}} \xi\right)$. To analytically solve the coupled equation system Eqs. (4.13) and (4.14), we decompose complex amplitudes $\mathrm{u}_{1}$ and $\mathrm{u}_{2}$ into absolute amplitudes and phase terms such as $\mathrm{u}_{1}=\mathrm{v}_{1} \exp \left(\mathrm{i} \phi_{1}\right)$ and $\mathrm{u}_{2}=\mathrm{v}_{2} \exp \left(\mathrm{i} \phi_{2}\right)$, therefore, the equation system Eqs. (4.13) and (4.14) gives four real differential equations. Solving these four equations we obtain the power and phase of a pulse remaining in each guide as it propagates along the coupler. Then pumping only one core by an external source of light, such that $\mathrm{v}_{1}(0, \tau)=\mathrm{v}_{2}(0, \tau)$, we get 


$$
\begin{gathered}
\mathrm{P} 2(\xi, \tau)=\frac{\mathrm{P}(0, \tau)}{4}\left(\frac{1}{\mathrm{p}_{1}}+\frac{1}{\mathrm{p}_{2}}-\frac{2 \cos (\Delta \phi)}{\sqrt{\mathrm{p}_{1} \mathrm{p}_{2}}}\right) \mathrm{e}^{-2 \alpha_{\llcorner N \xi}} \\
\Delta \phi(\xi, \tau)=2 \kappa \xi+\frac{\operatorname{Re}\left\{\mathrm{C}_{1}\right\}}{2 \operatorname{Im}\left\{\mathrm{C}_{1}\right\}} \ln \left(\mathrm{p}_{1}\right)-\frac{\operatorname{Re}\left\{\mathrm{C}_{2}\right\}}{2 \operatorname{Im}\left\{\mathrm{C}_{2}\right\}} \ln \left(\mathrm{p}_{2}\right)
\end{gathered}
$$

where $\mathrm{P} 2=\left|a_{2}\right|^{2} / \mathrm{P}_{0}$ is the normalized power coming out of the output notpumping port, $\quad \mathrm{p}_{1}=1+\left(\operatorname{Im}\left\{\mathrm{C}_{1}\right\} / 4 \gamma_{0}\right) \mathrm{P}(0, \tau) \mathrm{L}_{\text {eff }}(2 \xi) \quad$ and $\mathrm{p}_{2}=1+\left(\operatorname{Im}\left\{\mathrm{C}_{2}\right\} / 4 \gamma_{0}\right) \mathrm{P}(0, \tau) \mathrm{L}_{\text {eff }}(2 \xi)$. The phase difference and the effective length are given by $\Delta \phi(\xi, \tau)=\phi_{1}(\xi, \tau)-\phi_{2}(\xi, \tau)$ and $\mathrm{L}_{\text {eff }}(2 \xi)=\left(1-\mathrm{e}^{-2 \alpha_{L N \xi}}\right) / \alpha_{\mathrm{LN}}$ , respectively. A similar relation like Eq. (4.15) can be deduced for $\mathrm{P} 1=\left|a_{1}\right|^{2} / \mathrm{P}_{0}$. For simplicity, in this work we assume that the input pulses have a Gaussian shape with the normalized power profile $P(0, \tau)=\exp \left(-\tau^{2}\right)$. Before solutions describe the evolution of the electric field along the nonlinear coupler. Also, they show how powers and phase difference are affected by the TPA. In fact, effects of TPA on nonlinear pulse propagation already have been demonstrated for one silicon waveguide [6]. In the most general form, results indicate that power-dependent coupling terms (i.e. terms involving $\mathrm{Q}_{3}$ ), in conjunction with the TPA coefficient $r$, affect considerably the propagation of pulses along the coupler. Specifically, the solutions show the output power as a function of the linear losses, the TPA coefficient, the power-dependent coupling term and the input pulse peak power given by $\alpha$, $r Q_{3}$ and $P_{0}$, respectively. In the limit $Q_{3} \rightarrow 0$ and $r \rightarrow 0$ (no powerdependent coupling coefficients and no TPA), we get back the standard results used for fiber couplers [7]. The form of the phase difference is another important result because it deviates from the standard definition. The terms including $\mathrm{Q}_{3}$ have a considerably weight in changing the phase 
difference. In the limit $Q_{3} \rightarrow 0$, we get a parallel result used for silicon nanowires [6]. Therefore, if we take into account the power-dependent coupling effect incorporated into $Q_{3}$, the standard definition of the coupling length $\mathrm{L}_{\mathrm{c}}$ must changes.

To find transmittance as a function of the input pulse total power $P_{0}$ at the two output ports of the nonlinear coupler of length $L=L_{c}$, we use the following energy transmission coefficient

$$
\mathrm{T}_{\mathrm{n}}(\xi)=\frac{1}{\Omega} \int_{-\infty}^{\infty}\left|a_{\mathrm{n}}(\xi, \tau)\right|^{2} \mathrm{~d} \tau
$$

where $n=1,2$, depending of the output port that we are investigating. Here

$$
\Omega(\xi)=\int_{-\infty}^{\infty}\left(\left|a_{1}(\xi, \tau)\right|^{2}+\left|a_{2}(\xi, \tau)\right|^{2}\right) \mathrm{d} \tau
$$

represents the total energy and because the presence losses in the coupled nonlinear equations, $\Omega$ changes along $\xi$.

To illustrate the switching behavior in this case and along the rest of the work, we will focus on a silicon nanowire based nonlinear directional coupler in which the individual waveguides have the following dimensions: $\mathrm{W}=860 \mathrm{~nm}, \mathrm{H}=400 \mathrm{~nm}$ and $\mathrm{h}=300 \mathrm{~nm}$ [8]. The core-to-core separation distance can be taken such that $\kappa_{2}=957 \mathrm{~m}^{-1}$, initially. However, over all the work the core-to-core separation distance $S$ is changed to analyze the switching process. Also, we use the following parameters in the model: $\mathrm{n}_{2}=6 \times 10^{-5} \mathrm{~cm}^{2} / \mathrm{GW}, \quad \alpha=5 \mathrm{~dB} / \mathrm{cm}, \quad \beta_{2}=-2.15 \mathrm{ps}^{2} / \mathrm{m}, \quad \lambda_{0}=1484 \mathrm{~nm}$, $A_{\text {eff }}=0.13 \mu \mathrm{m}^{2}, r=0.1$ [8] and $\tau_{0}=1 \mathrm{~ns}$ [6]. To simplify our illustration at the moment, we take $\mathrm{Q}_{1}=\gamma_{0}(1+\mathrm{ir})$, where $\gamma_{0}=2 \pi \mathrm{n}_{2} / \lambda_{0} A_{\text {eff }}=195.4 \mathrm{~W}^{-1} / \mathrm{m}$, and the power-dependent coupling parameter can be represented by 
$\mathrm{dQ} \equiv \mathrm{Q}_{3} / \mathrm{Q}_{1}=0.05$. Figure 6 shows the evolution of the pulses along our nonlinear directional coupler with $L_{D}=46.5 \mathrm{~cm}$ and $\kappa_{2}=942 \mathrm{~m}^{-1}$. Initially, the nonlinear length for $P_{0}=4 \mathrm{~W}$ is given by $L_{N L}=1.3 \mathrm{~mm}$.

(a)
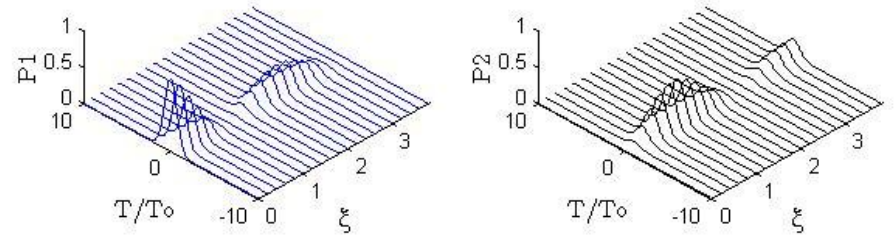

(b)
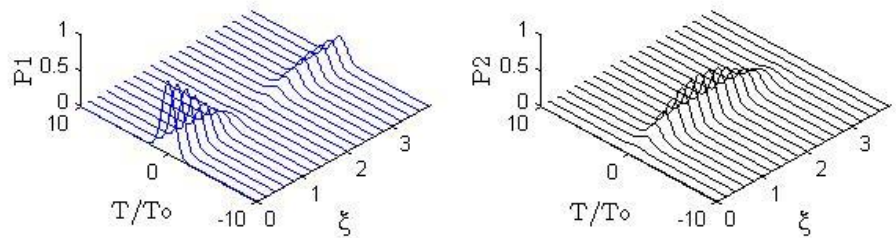

(c)
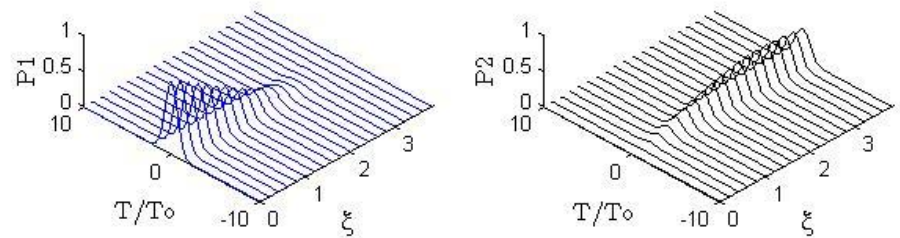

Figure 6. Evolution of pulses inside the nonlinear directional silicon coupler for solitonlike input with $T_{0}=1 \mathrm{ps} \kappa=1.22, Q_{3} / Q_{1}=0.05$ and $L=L_{C}$ : (a) $P_{0}=4 \mathrm{~W}$; (b) $P_{0}=6 \mathrm{~W} ;(c) P_{0}=12 \mathrm{~W}$.

To investigate the transmission characteristics at the two output ports of the nonlinear coupler of length $L=L_{c}$, we need to find out the transmission coefficient $T_{n}(\xi)$. Particularly, for the case of the output not-pumping port, transmission is given by

$$
\mathrm{T}_{2}(\xi)=\frac{1}{2}-\int_{-\infty}^{\infty} \frac{\mathrm{P}(0, \tau)}{\sqrt{\mathrm{p}_{1} \mathrm{p}_{2}}} \cos (\Delta \phi) \mathrm{d} \tau / \int_{-\infty}^{\infty} \frac{\left(\mathrm{p}_{1}+\mathrm{p}_{2}\right)}{\mathrm{p}_{1} \mathrm{p}_{2}} \mathrm{P}(0, \tau) \mathrm{d} \tau
$$


In Figure 7, we show the switching transmission characteristics in the limit $\mathrm{Q}_{3} \rightarrow 0$. The periodic behavior of output powers is appreciated for a range of $0-120 W$, approximately.

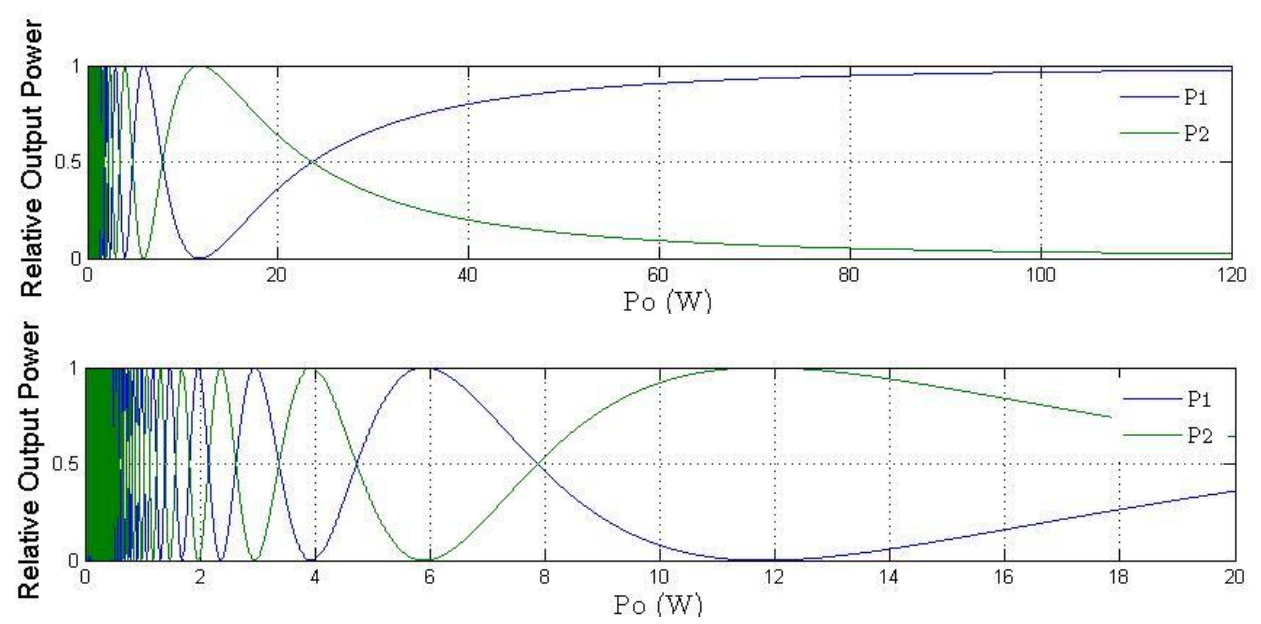

Figure 7. Switching characteristics for a silicon nanowire based nonlinear directional coupler of length $L=L_{C}$. Waveguides are separated at a distance $S$ such that $\kappa=1.22$ when $Q_{3}=0$. Relative output powers of ports 1 and 2 are shown as a function of input pulse power for 1 ps pulses.
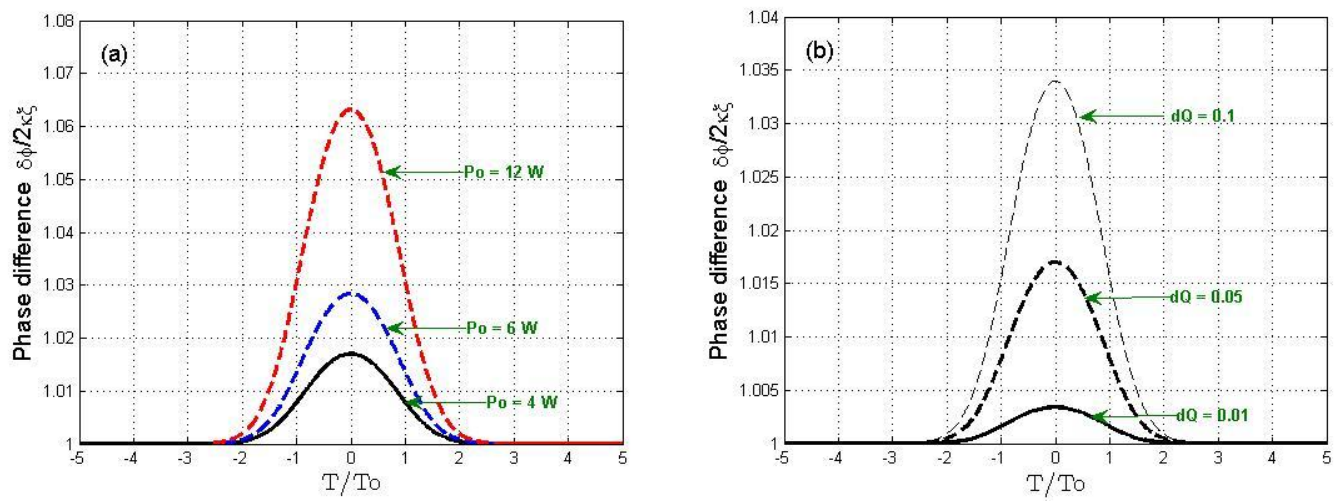

Figure 8. Phase difference of pulses in the nonlinear directional silicon coupler for solitonlike input with $T_{o}=1 \mathrm{ps}, \kappa=1.22$ and $L=L_{C}$ : (a) Phase difference for different input powers at $Q_{3} / Q_{1}=0.05$; (b) Phase difference for different values of $d Q=Q_{3} / Q_{1}$. 


\subsubsection{Free-Carrier Effects}

Since the TPA-induced free-carrier density $\bar{N}$ has a profound effect on the pulse propagation in silicon nanowires, therefore, it is desirable to construct an analytical description of the nonlinear evolution of optical pulses inside coupled silicon nanowires that includes the impact of free carriers. For this purpose, we derive the coupled evolution equations that describe the behavior of pulse envelopes, $a_{1}$ and $a_{2}$, on the nonlinear coupler under realistic situations. In this way, the coupled nonlinear equations can be represented by

$$
\begin{aligned}
& \frac{\partial \mathrm{u}_{1}}{\partial \xi}=i \kappa \mathrm{u}_{1}+i \mathrm{e}^{-2 \alpha_{\mathrm{LN} \xi}} \frac{\mathrm{C}_{1}}{\gamma_{0}} \mathrm{u}_{1}\left|\mathrm{u}_{1}\right|^{2}-\frac{\sigma}{2}(1+\mathrm{i} \mu) \mathrm{L}_{\mathrm{NL}} \overline{\mathrm{N}} \mathrm{u}_{1} \\
& \frac{\partial u_{2}}{\partial \xi}=-i \kappa u_{2}+i e^{-2 \alpha_{L N} \xi} \frac{C_{2}}{\gamma_{0}} u_{2}\left|u_{2}\right|^{2}-\frac{\sigma}{2}(1+i \mu) L_{N L} \bar{N} u_{2}
\end{aligned}
$$

where the new variables are given by $U_{1}=u_{1} \exp \left(-\alpha_{L N} \xi\right)$ and $\mathrm{U}_{2}=\mathrm{u}_{2} \exp \left(-\alpha_{\mathrm{LN}} \xi\right)$. Here, again, we have neglected the dispersive terms because we are assuming that the coupler length is much shorter than the dispersion length $L_{D}$. Therefore, the latter terms in each one of the coupledmode equations represent the effect of free carriers on the pulse propagation along the coupler. They give the change of the mode propagation constant by the free-carriers effect. As we have mentioned in the previous chapter, free carriers created by TPA are assumed to have equal densities, i.e., $N_{e}=N_{h} \equiv N$. In general, free carriers have a specific transverse density distribution inside the waveguides that form the coupler, but we can simplify the model by assuming that the free-carriers polarization is proportional to the carrier densities and so replacing $N$ with its averaged value $\bar{N}$ defined in Eq. (3.30). Equations (4.20) and (4.21) must be coupled to the equation that 
govern the evolution of the averaged free carriers density $\bar{N}$ over the optical mode profile, which are given by [10]

$$
\frac{\partial \bar{N}}{\partial \tau}=\frac{2 P_{0}^{2} \beta_{\mathrm{T}} e^{-4 \alpha_{\mathrm{L} \xi \xi}}}{h \omega_{0} A_{\text {eff }}^{2}}\left(\left|u_{1}\right|^{2}+\left|u_{2}\right|^{2}\right)^{2}-\frac{\bar{N}}{\tau_{0}}
$$

where $\tau_{0}$ is the carrier lifetime and we are assumed that effective mode area, $A_{\text {eff }}$, is nearly the same for the two waveguides, i.e., $A_{\text {eff }}^{(1)}=A_{\text {eff }}^{(2)}=A_{\text {eff }}$. In this regime, $\mathrm{u}_{1}$ and $\mathrm{u}_{2}$ are functions of propagation distance only. As a consequence, free-carriers density can be obtained from (4.22) by setting $\partial \overline{\mathrm{N}} / \partial \tau=0$ and is given by

$$
\overline{\mathrm{N}}(\mathrm{z})=\frac{2 \mathrm{P}_{0}^{2} \beta_{\mathrm{T}} \tau_{0} \mathrm{e}^{-4 \alpha_{\mathrm{L} \mathrm{k}}}}{\mathrm{h} \omega_{0} \mathrm{~A}_{\text {eff }}^{2}}\left(\left|\mathrm{u}_{1}\right|^{2}+\left|\mathrm{u}_{2}\right|^{2}\right)^{2}
$$

Now we follow a similar treatment using in [9] to obtain the solutions of the system. Therefore, using Eq. (4.23) into Eqs. (4.20) and (4.21), and introducing the new forms of supermodes waves, $u_{1}=\sqrt{P_{u 1}} \exp \left(i \phi_{1}\right)$ and $\mathrm{u}_{2}=\sqrt{\mathrm{P}_{\mathrm{u} 2}} \exp \left(\mathrm{i} \phi_{2}\right)$, we get

$$
\begin{aligned}
& \frac{\partial P_{\mathrm{u} 1}}{\partial \xi}=-\frac{2}{\gamma_{0}} \operatorname{Im}\left\{C_{1}\right\} e^{-2 \alpha_{L N} P_{u}^{2}} \mathrm{P}^{2}-2 \operatorname{Im}\{Z\} \mathrm{e}^{-4 \alpha_{\llcorner\sim} \xi} \mathrm{P}_{\mathrm{u} 1}\left(\mathrm{P}_{\mathrm{u} 1}+\mathrm{P}_{\mathrm{u} 2}\right)^{2}
\end{aligned}
$$

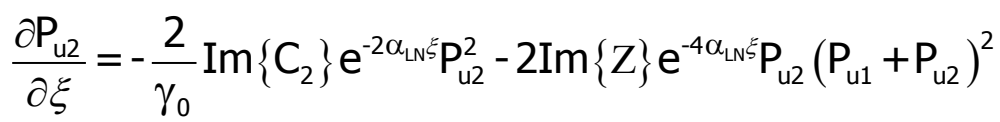

where $Z=-(\mu-i) \sigma L_{N L} P_{0}^{2} \beta_{T} \tau_{0} / h \omega_{0} A_{\text {eff }}^{2}$. In order to solve Eqs. (4.24) and (4.25) analytically, we make one reasonable simplification [9]. We discard first terms on the right side of Eqs. (4.24) and (4.25) since TPA is known to be much smaller than FCA in the case of long pulses [10]. With this simplification, solutions of Eqs. (4.24) and (4.25) are given by 


$$
\begin{aligned}
& \mathrm{P} 2(\xi, \tau)=\frac{\mathrm{P}(0, \tau)}{2} \frac{[1-\cos (\Delta \phi)] \mathrm{e}^{-2 \alpha_{\llcorner\perp \xi \xi}}}{\sqrt{1+4 \operatorname{Im}\{\Lambda\} \mathrm{P}^{2}(0, \tau) \mathrm{L}_{\text {eff }}(4 \xi)}} \\
& \Delta \phi(\xi, \tau)=2 \kappa \xi-\frac{\operatorname{Re}\left\{\mathrm{Q}_{3}\right\}}{\gamma_{0} \sqrt{\operatorname{Im}\{\Lambda\} \alpha_{\mathrm{LN}}}}\left[\arcsin \left(\frac{\mathrm{e}^{-2 \alpha_{\mathrm{LN}} \xi}}{\mathrm{A}}\right)-\arcsin \left(\frac{1}{\mathrm{~A}}\right)\right]
\end{aligned}
$$

where the input pulse is given by $\mathrm{P}(0, \tau)=4 \mathrm{P}_{\mathrm{u} 1}(0, \tau)$, which is a direct consequence of the initial conditions. The additional parameters are given by: the phase difference $\Delta \phi(\xi, \tau)=\phi_{1}(\xi, \tau)-\phi_{2}(\xi, \tau)$, this case effective length $\mathrm{L}_{\text {eff }}(4 \xi)=\left(1-\mathrm{e}^{-4 \alpha_{\mathrm{LN}} \xi}\right) / \alpha_{\mathrm{LN}}, \quad \mathrm{A}=\sqrt{\alpha_{\mathrm{LN}}+\operatorname{Im}\{\Gamma\} \mathrm{P}^{2}(0, \tau)} / \sqrt{\operatorname{Im}\{\Gamma\} \mathrm{P}^{2}(0, \tau)} \quad$ and $\operatorname{Im}\{\Gamma\}=\sigma L_{N L} P_{0}^{2} \beta_{T} \tau_{0} / h \omega_{0} A_{\text {eff }}^{2}$.

(a)
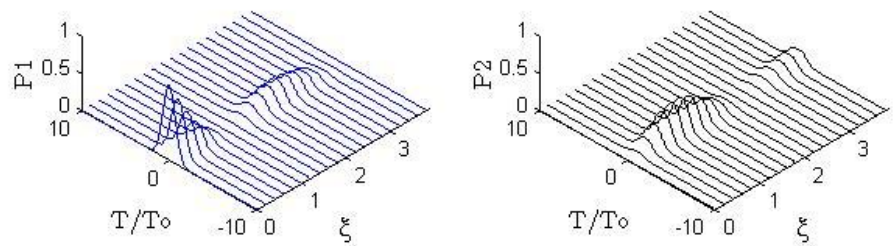

(b)
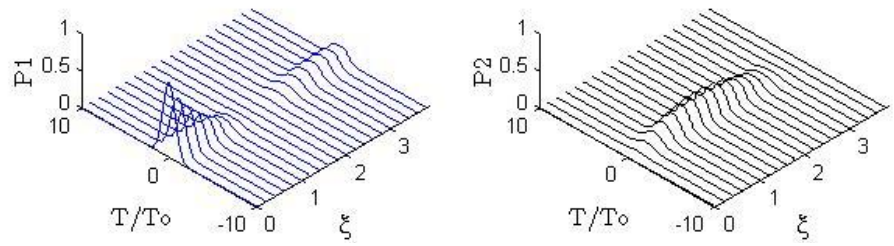

(c)
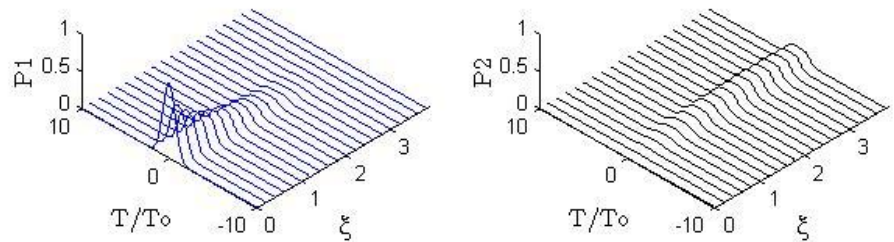

Figure 9. Evolution of pulses inside the nonlinear directional silicon coupler for solitonlike input with $T_{o}=10 \mathrm{ps} \kappa=1.22, Q_{3} / Q_{1}=0.05$ and $L=L_{C}$ : (a) $P_{0}=4 \mathrm{~W}$; (b) $P_{0}=6 \mathrm{~W} ;(c) P_{0}=12 \mathrm{~W}$. 
For simplicity, we have assumed again that the input pulses have a Gaussian shape with the normalized power profile $P(0, \tau)=\exp \left(-\tau^{2}\right)$. Figure 9 shows the evolution of the pulses along our nonlinear directional coupler with $L_{D}=46.5 \mathrm{~m}$ and $\kappa_{2}=942 \mathrm{~m}^{-1}$. For the case of the output not-pumping port, transmission is given by

$$
\mathrm{T}_{2}(\xi)=\frac{1}{2}-\frac{1}{2} \frac{\int_{-\infty}^{\infty} \frac{\mathrm{P}(0, \tau) \cos (\Delta \phi) \mathrm{d} \tau}{\sqrt{1+\operatorname{Im}\{\Lambda\} \mathrm{P}^{2}(0, \tau) \mathrm{L}_{\text {eff }}(4 \xi)}}}{\int_{-\infty}^{\infty} \frac{\mathrm{P}(0, \tau) \mathrm{d} \tau}{\sqrt{1+\operatorname{Im}\{\Lambda\} \mathrm{P}^{2}(0, \tau) \mathrm{L}_{\text {eff }}(4 \xi)}}}
$$

In the limit $\mathrm{Q}_{3} \rightarrow 0$, the switching transmission characteristics are the same like section 4.1 .1 for the same approximation.
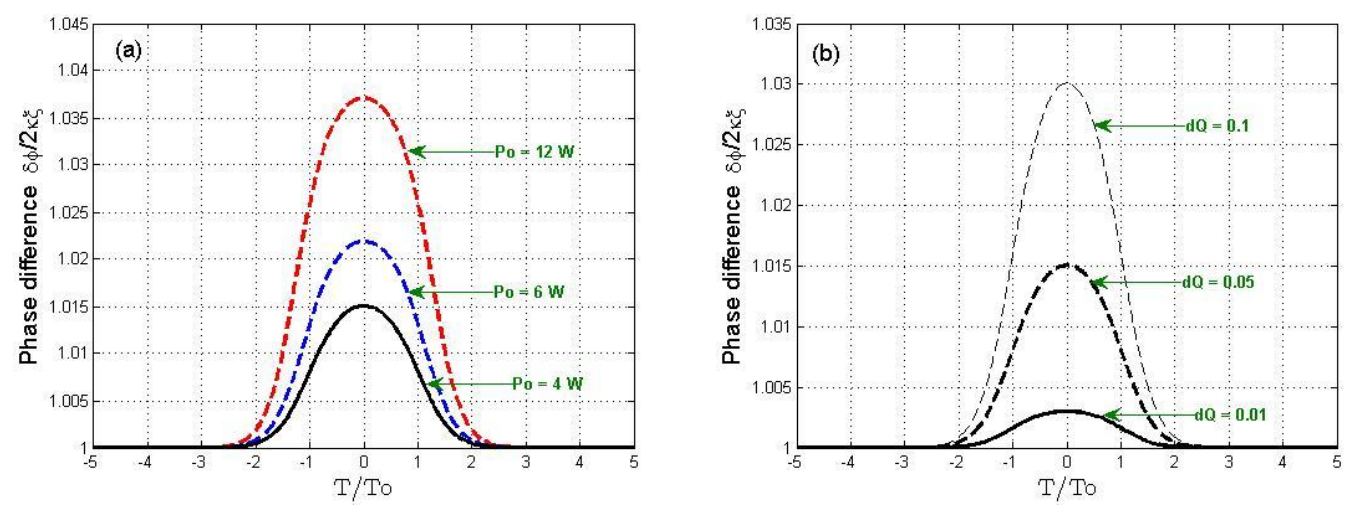

Figure 10. Phase difference of pulses in the nonlinear directional silicon coupler for solitonlike input with $T_{o}=10 \mathrm{ps}, \kappa=1.22$ and $L=L_{C}$ : (a) Phase difference for different input powers at $Q_{3} / Q_{1}=0.05 ;(b)$ Phase difference for different values of $d Q$. 


\subsubsection{Dispersion Effects}

Up to now, we have put forward the mathematical model that describes the passive coupler with nonlinearity and dispersion. Now we will assume that the coupler length $L$ is larger than the nonlinear length $L_{N L}$ and the dispersion length $L_{D}$. Therefore the equations system, describing the nonlinear coupling of dispersive waves inside the coupler, can be described like a special case of Eqs. (4.10) and (4.11) in a normalized form as:

$$
\begin{aligned}
& \frac{\partial \mathrm{U}_{1}}{\partial \xi}+\mathrm{i} \frac{\operatorname{sgn}\left(\beta_{2}\right)}{2 \mathrm{~N}^{2}} \frac{\partial^{2} \mathrm{U}_{1}}{\partial \tau^{2}}+\left(\alpha_{\mathrm{LN}}-\mathrm{i} \kappa\right) \mathrm{U}_{1}=\mathrm{i} \frac{\mathrm{C}_{1}}{\gamma_{0}} \mathrm{U}_{1}\left|\mathrm{U}_{1}\right|^{2} \\
& \frac{\partial \mathrm{U}_{2}}{\partial \xi}+\mathrm{i} \frac{\operatorname{sgn}\left(\beta_{2}\right)}{2 \mathrm{~N}^{2}} \frac{\partial^{2} \mathrm{U}_{2}}{\partial \mathrm{T}^{2}}+\left(\alpha_{\mathrm{LN}}+\mathrm{i} \kappa\right) \mathrm{U}_{2}=\mathrm{i} \frac{\mathrm{C}_{2}}{\gamma_{0}} \mathrm{U}_{2}\left|\mathrm{U}_{2}\right|^{2}
\end{aligned}
$$

where $\xi$ and $\tau$ represent the normalized distance and time variables previously defined in section 4.1. This physical model is based on generalized coupled Schrodinger equations where dispersion properties are given by the group velocity dispersion (GVD). The combination of $U_{1}$ and $U_{2}$ provides us with the general solutions for the envelopes $a_{1}$ and $a_{2}$ in the domain of picoseconds propagation pulses. In other words, $a_{2}=\sqrt{P_{0}}\left(U_{1}-U_{2}\right) \exp \left(i \kappa_{1} z\right)$, and $a_{1}=\sqrt{P_{0}}\left(\mathrm{U}_{1}+\mathrm{U}_{2}\right) \exp \left(i \kappa_{1} \mathrm{z}\right)$. The latter is input in one channel of the passive device to obtain the symmetric state. In order to solve the equations system, Eqs. (4.29) and (4.30), we take as the initial condition an arbitrary localized input and we use the entire scheme as a dissipative system. The corresponding derived equations account with the well known GinzburgLandau equation (CGLE) form with complex coefficients. In terms of the normalized power $\mathrm{P} 2=\left|a_{2}\right|^{2} / \mathrm{P}_{0}$, the solution becomes 


$$
\mathrm{P} 2(\zeta, \tau)=\operatorname{sech}^{2}\left(\mathrm{~K}_{1} \tau\right)[1-\cos (\Delta \Omega \xi)]
$$

where

$$
\begin{gathered}
\Delta \Omega=2 \kappa+\frac{\alpha_{L N}}{2} \frac{\left(1-\rho_{2}^{2}\right)}{\rho_{2}}-\frac{\alpha_{L N}}{2} \frac{\left(1-\rho_{1}^{2}\right)}{\rho_{1}}, \quad K_{v}^{2}=\frac{N^{2} \alpha_{L N}}{\rho_{v} \operatorname{sgn}\left(\beta_{2}\right)} \\
\rho_{v}=-3 \frac{\operatorname{Re}\left\{C_{v}\right\}}{\operatorname{Im}\left\{C_{v}\right\}}-\left[2+\left(3 \frac{\operatorname{Re}\left\{C_{v}\right\}}{\operatorname{Im}\left\{C_{v}\right\}}\right)^{2}\right]^{1 / 2}, \quad U_{0 v}=\left[\frac{\alpha_{L N}}{2} \frac{\gamma_{0}\left(\rho_{v}^{2}-2\right)}{\rho_{v} \operatorname{Re}\left\{C_{v}\right\}}\right]^{1 / 2}
\end{gathered}
$$

and the incident pulse has the hyperbolic secant form given by $P(0, \tau)=\operatorname{sech}^{2}\left(K_{1} \tau\right),\left|U_{01}\right|^{2}=1 / 2$, where $v=1$ or 2 . The previous solution shows that the pulse maintains its shape along the propagation but its peak power is modulated along the coupler. This latter characteristic is related to the exchange of power between waveguides that form the nonlinear coupler. Also, the preceding solution is subject to the boundary condition that a single beam is incident on one of the input ports such that $a_{1}(0, \tau)=2 \sqrt{P_{0} \mathrm{U}_{01}}\left[\operatorname{sech}\left(\mathrm{K}_{1} \tau\right)\right]^{1+i_{1}}$ and $a_{2}(0, \tau)=0$. A consequence of this initial condition, for the symmetric state, is $Q_{3}=0$. But Eq. (4.31) is a consequence of the superposition of two identical solitary pulse solutions to describe the behavior of field envelopes inside the nonlinear coupler. Figure 11 shows the initial conditions generated by the switching case: (a) shows how the input pulse broaden by reducing the input total power; (b) shows the relationship between the input total power and the total linear losses. The evolution of the pulses along the nonlinear coupler with $T_{0}=30$ fs for $P_{0}=4$, 6 and 12 W, is shown in Fig. 12. 

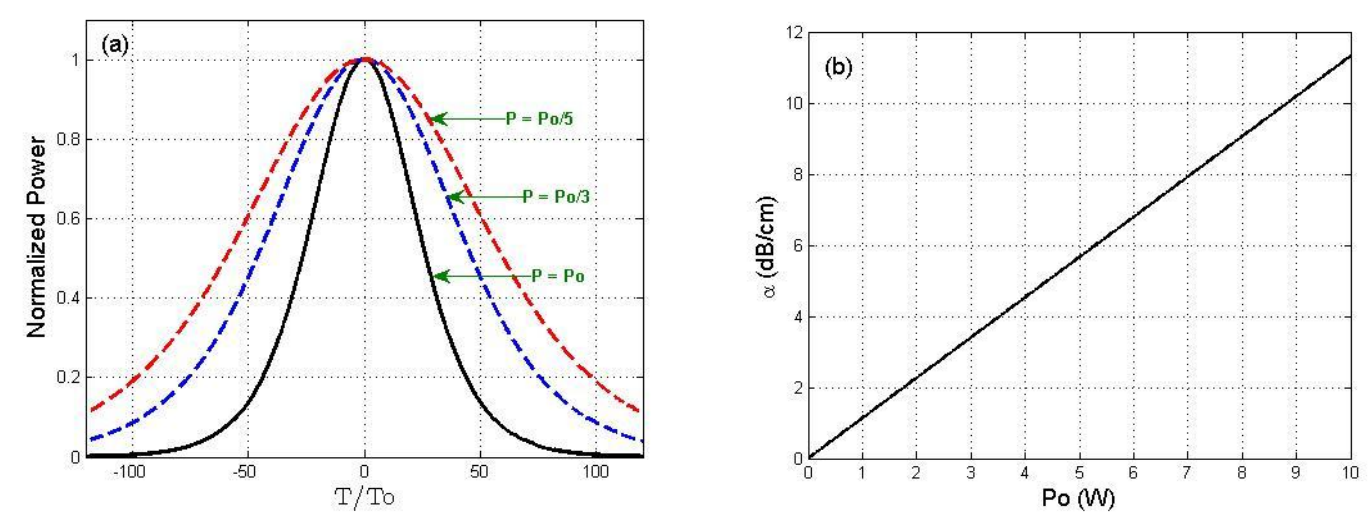

Figure 11. Initial conditions of the silicon nanowire based nonlinear directional coupler with $N=1$ : (a) shows the initial pulse shape for $P_{0}=12 \mathrm{~W}$ and $T_{0}=30 \mathrm{fs} ;$ (b) shows the relation between the input power and the propagation losses $\alpha$.

(a)
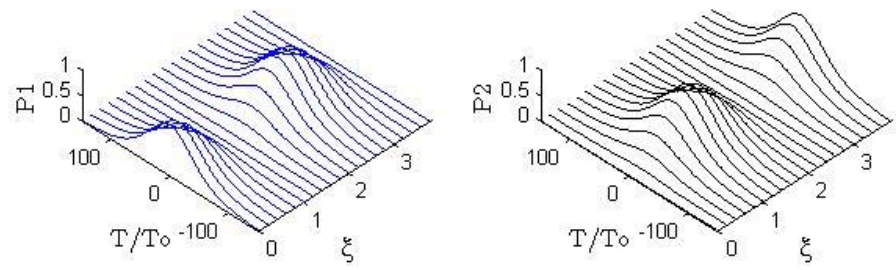

(b)
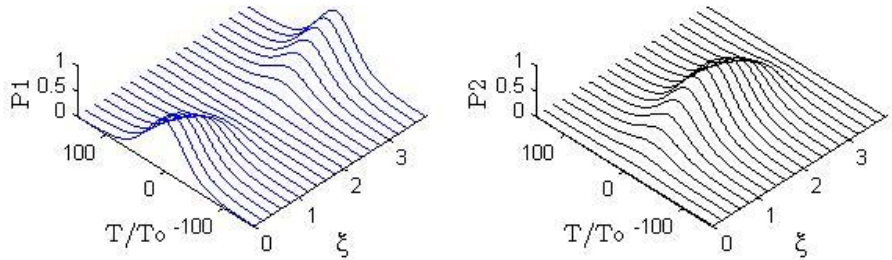

(c)
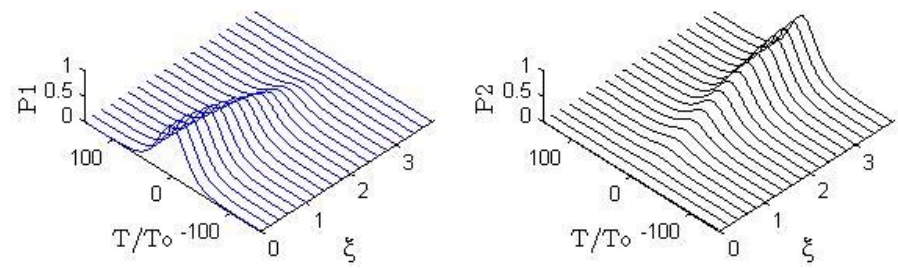

Figure 12. Evolution of pulses inside the nonlinear directional silicon coupler for solitonlike input with $T_{o}=30 \mathrm{ps} \kappa=1.22, Q_{3}=0$ and $L=5 \mathrm{~mm}$ : (a) $P_{0}=4 \mathrm{~W}$; (b) $P_{0}=6 \mathrm{~W}$ ; (c) $P_{0}=12 \mathrm{~W}$. 
In this case, the transmission coefficient $T_{2}(\xi)$ is given by

$$
\mathrm{T}_{2}(\xi)=\frac{1}{2}[1-\cos (\Delta \Omega \xi)]
$$

which gives the same result than Fig. 7 .

\subsection{Summary}

In this chapter, we have provided the analytical solutions for the coupledmode equations in those cases in which the density of the free carrier is relatively low, the dispersive effects are relatively weak and assuming that the nonlinear effects do not affect the waveguide modes significantly. The impact of two-photon absorption and free-carriers effect on the properties of the nonlinear coupling effects has been studied in detail together with the evolution of optical power inside an array of short silicon nanowires.

\section{References}

[1] Amarendra K. Sarma, "Silicon waveguide based nonlinear directional coupler as a soliton switch," Opt. Eng. 47, 120503 (Dec 12, 2008).

[2] Ivan D. Rukhlenko, Malin Premaratne, Chethiya Dissanayake, and Govind P. Agrawal, "Nonlinear Pulse Evolution in Silicon Waveguides: An Approximate Analytic Approach," J. Lightwave Technol. 27, 3241-3248 (2009)

[3] S. M. Jensen, "The nonlinear coherent coupler," IEEE J. Quantum Electron., vol. QE-18, pp. 1580-1583, 1982.

[4] G. P. Agrawal, Lightwave Technology: Components and Devices (Wiley, Hoboken, NJ, 2004). 
[5] J. Fraile-Pelaez, and G. Assanto., A. Opt. Vol. 29, No. 15, (1990).

[6] L. Yin and G. P. Agrawal, "Impact of two-photon absorption on self-phase modulation in silicon waveguides," Opt. Lett. 32, 2031-2033 (2007).

[7] G. P. Agrawal, Applications of Nonlinear Fiber Optics, (Academic Press, Elsevier, 2001), 2nd expanded ed. 2008.

[8] Jidong Zhang, Qiang Lin, Giovanni Piredda, Robert W. Boyd, Govind P. Agrawal, and Philippe M. Fauchet, "Optical solitons in a silicon waveguide," Opt. Express 15, 7682-7688 (2007).

[9] I. D. Rukhlenko, M. Premaratne, and G. P. Agrawal, "Nonlinear silicon photonics: Analytical tools," IEEE J. Sel. Top. Quantum Electron. 16, 200-215 (2010).

[10] Q. Lin, O. J. Painter, and G. P. Agrawal, "Nonlinear optical phenomena in silicon waveguides: Modeling and applications," Opt. Express 15, 1660416644 (2007). 


\section{Chapter 5}

\section{Conclusions}

In conclusion, we may say that a comprehensive theory was developed for describing the nonlinear optical propagation of short pulses in coupled silicon nanowires. A generalized analysis of the nonlinear coupled system was given where we have considered the vector nature of optical modes and the effects of TPA on various nonlinear processes. The general theoretical model included not only the effects of FCA and FCD but also linear and nonlinear losses, This model also extends previous vector nonlinear models to the case where coupling of supermodes of a waveguide array occurs in silicon waveguides. Although, there are some investigations already done in the literature about nonlinear propagation of optical pulses in silicon waveguides, the material presented here was deduced for the first time. This theoretical investigation was inspired by the work done by Agrawal, et. al about nonlinear propagation of pulses inside silicon waveguides and its applications. The crucial importance of this theoretical investigation on nonlinear effects in coupled silicon nanowires is related to the all-optical switching application.

It has been demonstrated theoretically that free carriers generated by TPA in silicon nanowires introduces large losses at telecommunications wavelengths. Two-photon absorption is a nonlinear optical loss mechanism derived of radiation matter-interaction in silicon waveguides at communications wavelengths. Two-photon absorption, free-carrier absorption, and free-carrier 
dispersion play important roles in the nonlinear propagation of optical pulses inside coupled silicon nanowires. They have been studied theoretically on the transmission of picoseconds optical pulses in silicon nanowires in this work. 


\section{Appendix A}

In this appendix we are going to derive the Eq. (3.11). For that, first we substitute Eq. (3.8) into Eq. (3.7), then multiply the resulting equation by $\mathbf{e}_{\mathrm{k}}^{*}$, and finally we integrate over the transverse dimensions and associate terms. Therefore, we obtain that the mode amplitudes satisfy

$$
\begin{aligned}
& \sum_{\mathrm{m}} \frac{\tilde{a}_{\mathrm{m}}}{\sqrt{\mathrm{N}_{\mathrm{m}}}} \iint \mathbf{e}_{\mathrm{k}}^{*} \cdot\left[\nabla_{\mathrm{T}}^{2} \mathbf{e}_{\mathrm{m}}-\nabla_{\mathrm{T}}\left(\nabla_{\mathrm{T}} \cdot \mathbf{e}_{\mathrm{Tm}}\right)\right] \mathrm{dxdy}+\sum_{\mathrm{m}} \frac{1}{\sqrt{\mathrm{N}_{\mathrm{m}}}}\left(\frac{\partial^{2} \tilde{a}_{\mathrm{m}}}{\partial \mathrm{z}^{2}}+2 \mathrm{i} \beta \frac{\partial \tilde{a}_{\mathrm{m}}}{\partial \mathrm{z}}-\beta^{2} \tilde{a}_{\mathrm{m}}\right) \\
& \quad \times \iint \mathbf{e}_{\mathrm{Tk}}^{*} \cdot \mathbf{e}_{\mathrm{Tm}} \mathrm{dxdy}-\sum_{\mathrm{m}} \frac{1}{\sqrt{\mathrm{N}_{\mathrm{m}}}} \frac{\partial \tilde{a}_{\mathrm{m}}}{\partial \mathrm{z}} \iint\left[\mathbf{e}_{\mathrm{Tk}}^{*} \cdot \nabla_{\mathrm{T}} \mathrm{e}_{\mathrm{zm}}+\mathrm{e}_{\mathrm{zk}}^{*}\left(\nabla_{\mathrm{T}} \cdot \mathbf{e}_{\mathrm{Tm}}\right)\right] \mathrm{dxdy} \\
& +\sum_{\mathrm{m}} \frac{\tilde{a}_{\mathrm{m}}}{\sqrt{\mathrm{N}_{\mathrm{m}}}} \frac{\omega^{2}}{\mathrm{c}^{2}} \iint \mathbf{e}_{\mathrm{k}}^{*} \mathrm{n}_{0}^{2} \mathbf{e}_{\mathrm{m}} \mathrm{dxdy}-\mathrm{i} \beta \sum_{\mathrm{m}} \frac{\tilde{a}_{\mathrm{m}}}{\sqrt{\mathrm{N}_{\mathrm{m}}}} \iint\left[\mathbf{e}_{\mathrm{Tk}}^{*} \cdot \nabla_{\mathrm{T}} \mathrm{e}_{\mathrm{zm}}+\mathrm{e}_{\mathrm{zk}}^{*}\left(\nabla_{\mathrm{T}} \cdot \mathbf{e}_{\mathrm{Tm}}\right)\right] \mathrm{dxdy} \\
& =-\mu_{0} \omega^{2} \mathrm{e}^{-i \beta z} \iint \mathbf{e}_{\mathrm{k}}^{*} \cdot\left[\tilde{\mathbf{P}}^{\mathrm{f}}+\tilde{\mathbf{P}}^{(3)}\right] \mathrm{dxdy},
\end{aligned}
$$

where the vectorial modes for the electric and magnetic fields were decompose into longitudinal and transverse components, parallel to and orthogonal to the waveguide axis, respectively, and denoted by subscripts $z$ and $\mathrm{T}$, where $\mathbf{e}_{\mathrm{m}}=\mathbf{e}_{\mathrm{Tm}}+\mathrm{e}_{\mathrm{zm}} \hat{\mathbf{z}} ; \mathbf{h}_{\mathrm{m}}=\mathbf{h}_{\mathrm{Tm}}+\mathrm{h}_{\mathrm{zm}} \hat{\mathbf{z}}$, and $\hat{\mathbf{z}}$ is the unit vector parallel to the waveguide axis. Also here, the asterisk denotes the complex conjugation. Using Eq. (3.10) in the extended form 


$$
\begin{aligned}
& \nabla_{\mathrm{T}}^{2} \mathbf{e}_{\mathrm{m}}-\beta_{\mathrm{m}}^{2} \mathbf{e}_{\mathrm{m}}+\mathrm{k}_{0}^{2} \mathrm{n}_{0 \mathrm{~m}}^{2} \mathbf{e}_{\mathrm{m}}-\nabla_{\mathrm{T}}\left(\nabla_{\mathrm{T}} \cdot \mathbf{e}_{\mathrm{Tm}}\right) \\
& \quad-\mathrm{i} \beta_{\mathrm{m}} \nabla_{\mathrm{T}} \mathrm{e}_{\mathrm{zm}}-\mathrm{i} \beta_{\mathrm{m}}\left(\nabla_{\mathrm{T}} \cdot \mathbf{e}_{\mathrm{Tm}}\right) \hat{\mathbf{z}}+\beta_{\mathrm{m}}^{2} \mathrm{e}_{\mathrm{zm}} \hat{\mathbf{z}}=0
\end{aligned}
$$

and making the slowly varying envelope approximation, Eq. (A.1) becomes

$$
\begin{aligned}
& \sum_{\mathrm{m}} \frac{1}{\sqrt{\mathrm{N}_{\mathrm{m}}}}\left[\frac{\partial \tilde{a}_{\mathrm{m}}}{\partial \mathrm{z}}-\mathrm{i}\left(\beta_{\mathrm{m}}-\beta\right) \tilde{a}_{\mathrm{m}}\right] \iint\left[2 \mathrm{i} \beta \mathbf{e}_{\mathrm{Tk}}^{*} \cdot \mathbf{e}_{\mathrm{Tm}}-\mathbf{e}_{\mathrm{Tk}}^{*} \cdot \nabla_{\mathrm{T}} \mathrm{e}_{\mathrm{zm}}-\mathrm{e}_{z \mathrm{k}}^{*}\left(\nabla_{\mathrm{T}} \cdot \mathbf{e}_{\mathrm{Tm}}\right)\right] \mathrm{dxdy} \\
& +\sum_{\mathrm{m}} \frac{\tilde{a}_{\mathrm{m}}}{\sqrt{\mathrm{N}_{\mathrm{m}}}} \frac{\omega^{2}}{\mathrm{c}^{2}} \iint \mathbf{e}_{\mathrm{k}}^{*}\left(\mathrm{n}_{0}^{2}-\mathrm{n}_{0 \mathrm{~m}}^{2}\right) \mathbf{e}_{\mathrm{m}} \mathrm{dxdy}=-\mu_{0} \omega^{2} \mathrm{e}^{-i \beta z} \iint \mathbf{e}_{\mathrm{k}}^{*} \cdot\left(\tilde{\mathbf{P}}^{\mathrm{f}}+\tilde{\mathbf{P}}^{(3)}\right) \mathrm{dxdy},
\end{aligned}
$$

where we have assumed that the light is spectrally narrow namely $\left|\omega-\omega_{0}\right| \square \omega_{0}$ or in the same way $\left|\beta_{\mathrm{m}}-\beta\right| \square \beta$. Hence the approximation $\beta_{\mathrm{m}}+\beta \approx 2 \beta$ is justified. The first integral in the last equation has been analyzed in [4] following the next form:

$$
\begin{aligned}
\iint & {\left[2 i \beta \mathbf{e}_{T k}^{*} \cdot \mathbf{e}_{T m}-\mathbf{e}_{T k}^{*} \cdot \nabla_{T} e_{z m}-e_{z k}^{*}\left(\nabla_{T} \cdot \mathbf{e}_{T m}\right)\right] d x d y } \\
& =i \omega \mu_{0} \iint\left(\mathbf{e}_{m} \times \mathbf{h}_{k}^{*}+\mathbf{e}_{k}^{*} \times \mathbf{h}_{m}\right) \cdot \hat{z} d x d y .
\end{aligned}
$$

With this equivalency, Eq. (A.3) becomes

$$
\begin{aligned}
& \sum_{\mathrm{m}} \frac{1}{\sqrt{\mathrm{N}_{\mathrm{m}}}}\left[\frac{\partial \tilde{a}_{\mathrm{m}}}{\partial \mathrm{z}}-\mathrm{i}\left(\beta_{\mathrm{m}}-\beta\right) \tilde{a}_{\mathrm{m}}\right] \iint\left(\mathbf{e}_{\mathrm{m}} \times \mathbf{h}_{\mathrm{k}}^{*}+\mathbf{e}_{\mathrm{k}}^{*} \times \mathbf{h}_{\mathrm{m}}\right) \cdot \hat{\mathbf{z}} \mathrm{dxdy} \\
& \quad=\mathrm{i} \omega \varepsilon_{0} \sum_{\mathrm{m}} \frac{\tilde{a}_{\mathrm{m}}}{\sqrt{\mathrm{N}_{\mathrm{m}}}} \iint \mathbf{e}_{\mathrm{k}}^{*} \Delta \mathrm{n}_{\mathrm{m}}^{2} \mathbf{e}_{\mathrm{m}} \mathrm{d} x \mathrm{dy}+\mathrm{i} \omega \mathrm{e}^{-\mathrm{i} \beta z} \iint \mathbf{e}_{\mathrm{k}}^{*} \cdot\left(\tilde{\mathbf{P}}^{\mathrm{f}}+\tilde{\mathbf{P}}^{(3)}\right) \mathrm{d} \mathrm{d} \mathrm{dy},
\end{aligned}
$$

where $\Delta n_{m}^{2}=n^{2}-n_{m}^{2}$ is the change in the refraction index due to the presence of the mth guide mode. This work will be consistent with the assumption of orthogonality, as follows 


$$
\iint\left(\mathbf{e}_{\mathrm{m}} \times \mathbf{h}_{\mathrm{k}}^{*}+\mathbf{e}_{\mathrm{k}}^{*} \times \mathbf{h}_{\mathrm{m}}\right) \cdot \hat{\mathbf{z}} \mathrm{dxdy}=4 \mathrm{~N}_{\mathrm{k}} \delta_{\mathrm{mk}}
$$

where $N_{k}$ is given in the Eq. (3.9). Therefore, substituting the above relation into Eq. (A.5) leads to the following nonlinear amplitude equation in the frequency domain

$$
\frac{\partial \tilde{a}_{\mathrm{k}}}{\partial \mathrm{z}}=\mathrm{i}\left(\beta_{\mathrm{k}}-\beta\right) \tilde{a}_{\mathrm{k}}+\mathrm{i} \sum_{\mathrm{m}} \kappa_{\mathrm{km}} \tilde{a}_{\mathrm{m}}+\frac{\mathrm{i} \omega \mathrm{e}^{-i \beta z}}{4 \sqrt{\mathrm{N}_{\mathrm{k}}}} \iint \mathbf{e}_{\mathrm{k}}^{*} \cdot\left(\tilde{\mathbf{P}}^{\mathrm{f}}+\tilde{\mathbf{P}}^{(3)}\right) \mathrm{dxdy},
$$

in which the parameter $\kappa_{\mathrm{km}}$ is the coupling coefficient and is given by

$$
\kappa_{\mathrm{km}}=\frac{\omega \varepsilon_{0}}{4 \sqrt{\mathrm{N}_{\mathrm{k}} \mathrm{N}_{\mathrm{m}}}} \iint \mathbf{e}_{\mathrm{k}}^{*} \Delta \mathrm{n}_{\mathrm{m}}^{2} \mathbf{e}_{\mathrm{m}} \mathrm{dxdy} .
$$

Note that Eq. (A.7) is the most general form that the mode amplitudes satisfy. In this equation no perturbation has been considered for dispersion and nonlinearity. The terms shown in this equation correspond to the dispersion, coupling and nonlinearity, respectively, into the right hand. 


\section{List of Figures}

Figure 1. Four configurations of waveguides in silicon: (a) Chanel waveguides; (b) Rib waveguides; (c) Photonic-crystal waveguides; (d) Slot waveguides. (Source: [9]).

Figure 2. Cross section and waveguide parameters of an SOI rib waveguide. (Source: [13]).

Figure 3. Calculated dispersion curves for the TE and TM modes. The design of their waveguide is shown in the inset (Source: [6]).

Figure 4. Nonlinear parameter $\gamma_{11}$ as a function of waveguide width and height at the $1550 \mathrm{~nm}$ wavelength (Source: [7]).

Figure 5. Coupled silicon nanowires design: (a) schematic illustration of the four-port device; (b) scheme of the cross-section.

Figure 6. Evolution of pulses inside the nonlinear directional silicon coupler for solitonlike input with $T_{0}=1 \mathrm{ps}, \kappa=1.22, \mathrm{Q}_{3} / \mathrm{Q}_{1}=0.05$ and $\mathrm{L}=\mathrm{L}_{\mathrm{C}}$ : (a) $\mathrm{Po}=4 \mathrm{~W}$; (b) Po $=6 \mathrm{~W}$; (c) $\mathrm{Po}=12 \mathrm{~W}$.

Figure 7. Switching characteristics for a silicon nanowire based nonlinear directional coupler of length $L=L_{C}$. Waveguides are separated at a distance $s$ such that $\kappa=1.22$ when $\mathrm{Q}_{3}=0$. Relative output powers of ports 1 and 2 are shown as a function of input pulse power for 1 ps pulses.

Figure 8. Phase difference of pulses in the nonlinear directional silicon coupler for solitonlike input with $T_{o}=1 \mathrm{ps}, \kappa=1.22$ and $L_{=} \mathrm{L}_{\mathrm{C}}$ : (a) Phase difference for different input powers at $Q_{3} / Q_{1}=0.05$; (b) Phase difference for different values of $d Q$. 
Figure 9. Evolution of pulses in the nonlinear directional silicon coupler for solitonlike input with $T_{0}=10 \mathrm{ps}, \kappa=1.22, \mathrm{Q}_{3} / \mathrm{Q}_{1}=0.05$ and $\mathrm{L}_{=} \mathrm{L}_{\mathrm{C}}$ : (a) $\mathrm{Po}=4 \mathrm{~W}$; (b) Po $=6 \mathrm{~W}$. ; (c) $\mathrm{Po}=12 \mathrm{~W}$.

Figure 10. Phase difference of pulses in the nonlinear directional silicon coupler for solitonlike input with $T_{0}=10 \mathrm{ps}, \kappa=1.22$ and $L=L_{C}$ : (a) Phase difference for different input powers at $\mathrm{Q}_{3} / \mathrm{Q}_{1}=0.05$; (b) Phase difference for different values of $d Q$.

Figure 11. Initial conditions of the silicon nanowire based nonlinear directional coupler $N=1$ : (a) shows the initial pulse shape for $P_{o}=12 \mathrm{~W}$ and $\mathrm{T}_{\mathrm{o}}=30 \mathrm{fs}$; (b) shows the relation between the input power and the propagation losses $\alpha$.

Figure 12. Evolution of pulses in the nonlinear directional silicon coupler for solitonlike input with $\mathrm{To}_{0}=30 \mathrm{fs}, \kappa=1.22, \mathrm{Q}_{3}=0$ and $\mathrm{L}=5 \mathrm{~mm}$ : (a) $P_{o}=4 W ;(b) P_{0}=6 W ;(b) P_{0}=12 W$. 